Supporting Information for:

\title{
Unsaturated Ligands Seed an Order to Disorder Transition in Mixed Ligand Shells of CdSe/CdS Quantum Dots
}

Arunima D. Balan,,$^{\dagger} \S$ Jacob H. Olshansky, ${ }^{\dagger}{ }^{\S}$ Yonatan Horowitz, ${ }^{\dagger}{ }^{\S}{ }^{\ddagger}$ Hui-Ling Han, ${ }^{\dagger}{ }^{\S}$ Erin A. O’Brien, ${ }^{\dagger}{ }^{\S}$ Lucio Tang, ${ }^{\dagger}$ Gabor A. Somorjai, ${ }^{\dagger}{ }^{\S}$ and A. Paul Alivisatos ${ }^{\dagger} \perp^{\S} * *$

$\dagger$ Department of Chemistry and \#Department of Materials Science and Engineering, University of California, Berkeley, California 94720, United States

$\perp$ Material Sciences Division, Lawrence Berkeley National Laboratory, Berkeley, California 94720, United States

$\S$ Kavli Energy NanoScience Institute, Berkeley, California 94720, United States

+ Y.H. Current Address: School of Chemistry Faculty of Exact Sciences, Tel Aviv University, Tel Aviv 6997801, Israel

Corresponding Author

*E-mail: paul.alivisatos@,berkeley.edu

\section{Table of Contents}

$\begin{array}{ll}\text { QD characterization } & \text { S2 }\end{array}$

$\begin{array}{ll}\text { QD absorption before and after ligand exchanges } & \text { S3 }\end{array}$

$\begin{array}{ll}\text { Calibrating the temperature } & \text { S4 }\end{array}$

$\begin{array}{ll}\text { Analyzing nonradiative and radiative rates vs. T } & \text { S8 }\end{array}$

$\begin{array}{ll}\text { Variable temperature IR data } & \text { S11 }\end{array}$

$\begin{array}{ll}\text { SFG data and analysis } & \text { S14 }\end{array}$

$\begin{array}{ll}\text { Concentration- and binding group-dependence of PL data } & \text { S17 }\end{array}$

$\begin{array}{ll}\text { Size scaling of the phase transition } & \text { S20 }\end{array}$

$\begin{array}{ll}\text { Cauchy distribution integrals to fit phase transition } & \text { S22 }\end{array}$

$\begin{array}{ll}\text { Ising model of phase transition } & \text { S25 }\end{array}$

$\begin{array}{ll}\text { Additional experimental methods } & \text { S27 }\end{array}$

$\begin{array}{lr}\text { References } & \text { S27 }\end{array}$ 


\section{QD characterization}

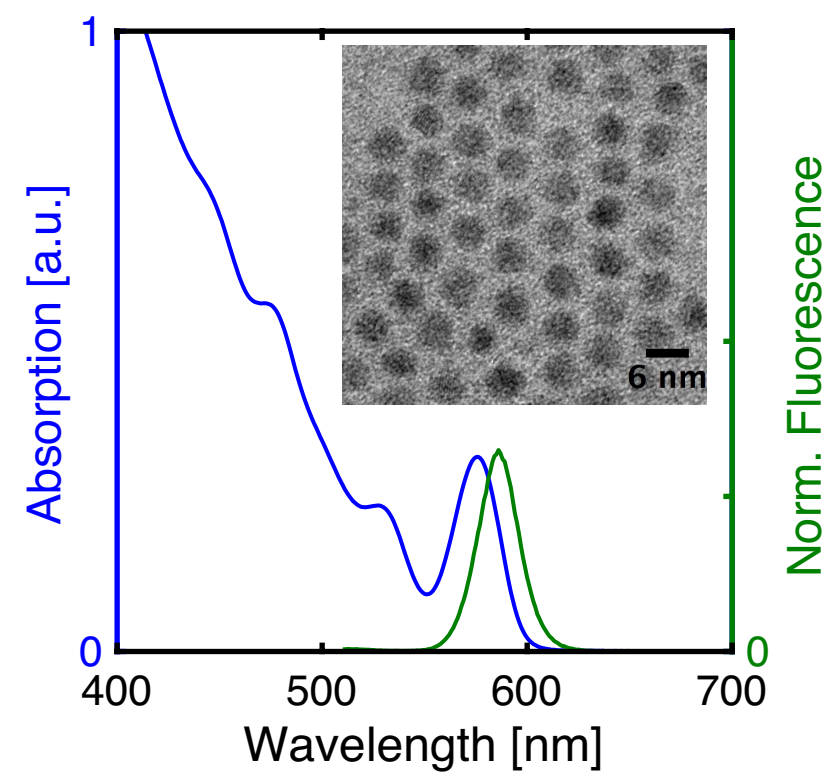

Figure S1: Absorption, emission and (inset: TEM) of QD sample. The average particle diameter is $4.95(+/-0.49) \mathrm{nm}$. 
QD absorption before and after ligand exchanges

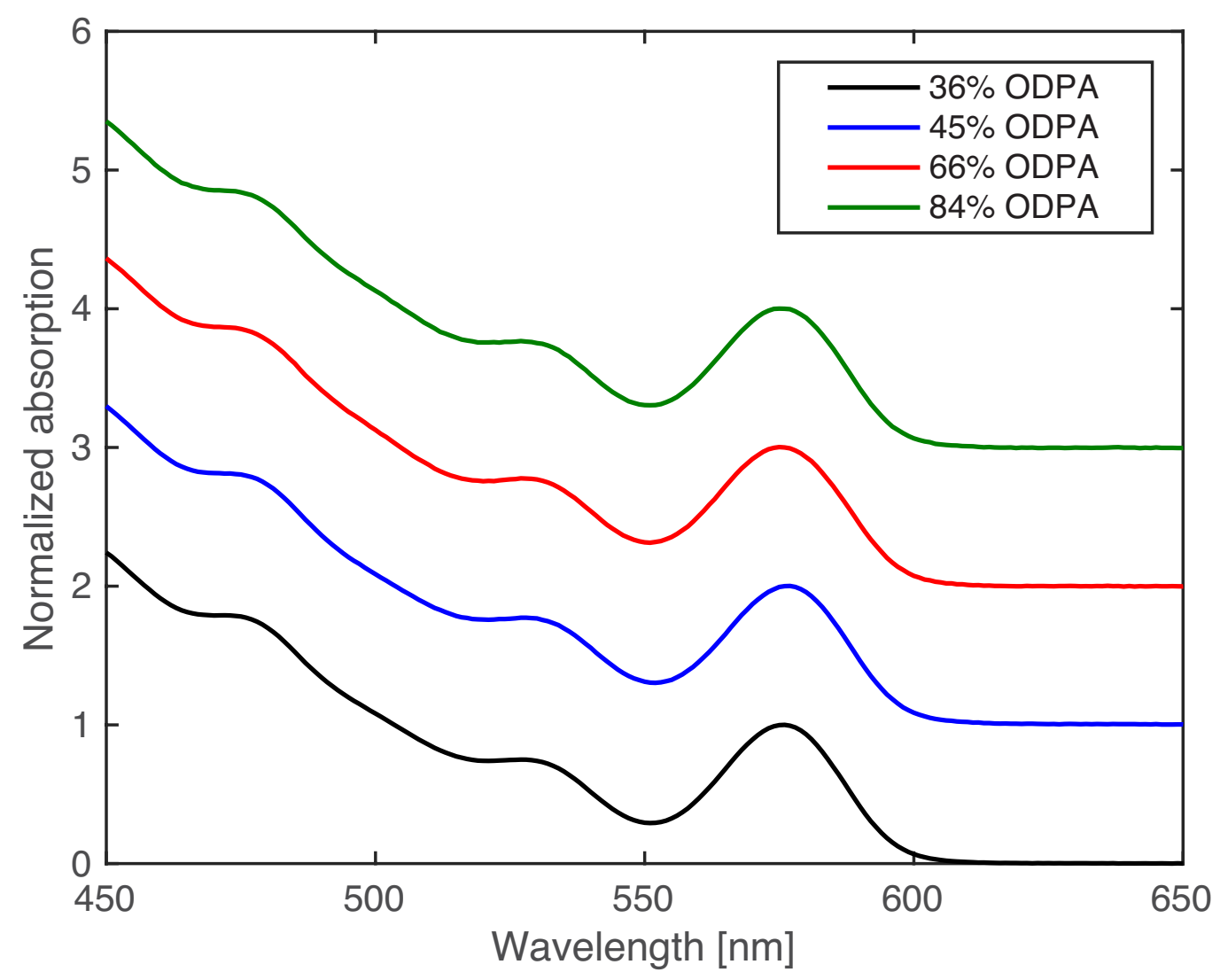

Figure S2: Absorption spectra (normalized to the first excitonic peak) of QD samples before and after ligand exchanges 
Calibrating the temperature
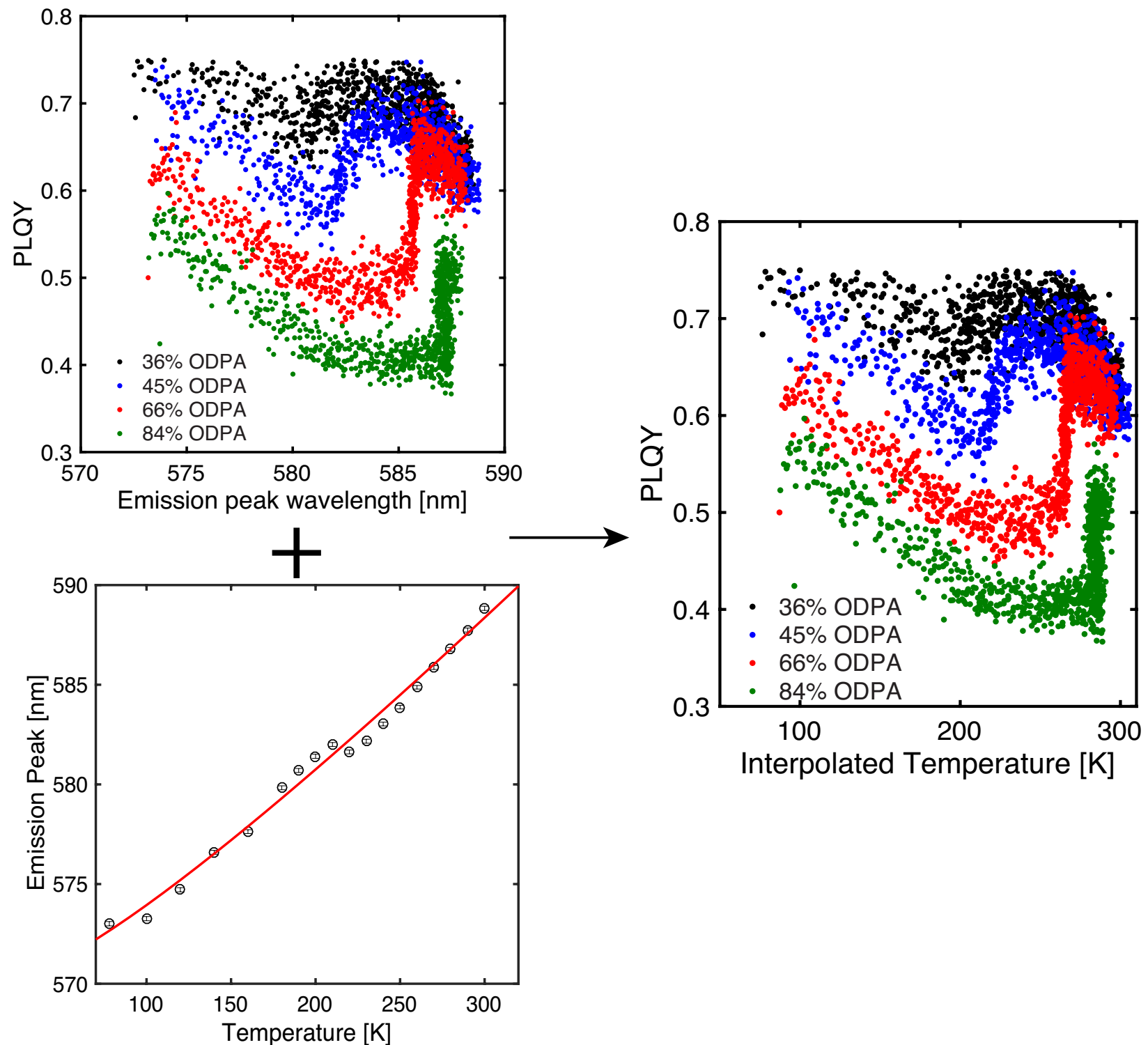

Figure S3: First step of the temperature calibration. The raw PLQY data are plotted against the emission peak wavelength. Temperature-dependent photoluminescence data collected in a cryostat on the $36 \%$ ODPA sample is used to calibrate the temperature. 

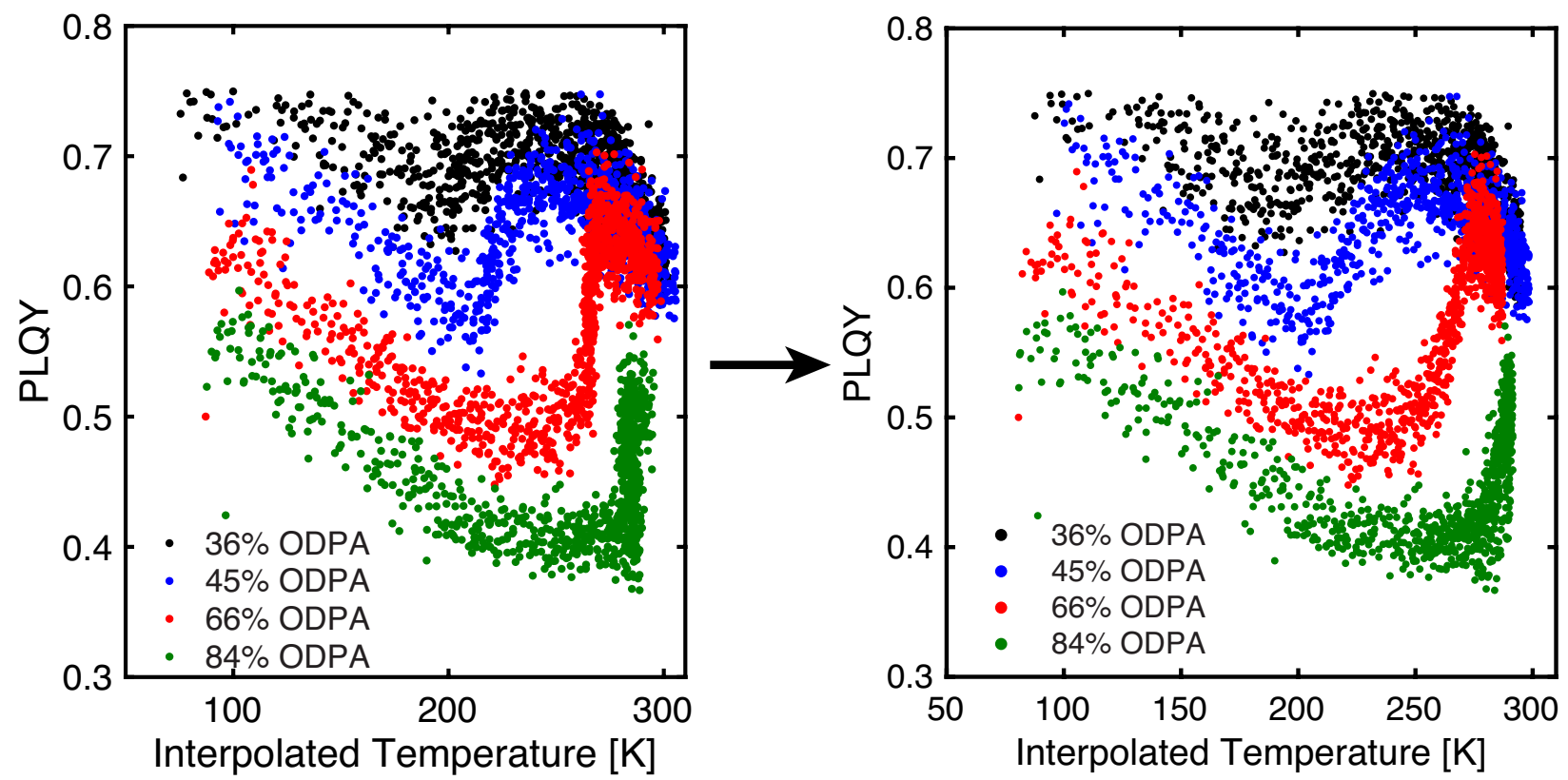

Figure S4: Second step of the temperature calibration. We use a Newton's law of cooling model to further process the temperature data. The results here show that it has minimal impact on the curves, except to somewhat stretch out the phase transition and to remove the apparent nonmonotonic behavior shown in the $84 \%$ ODPA sample.

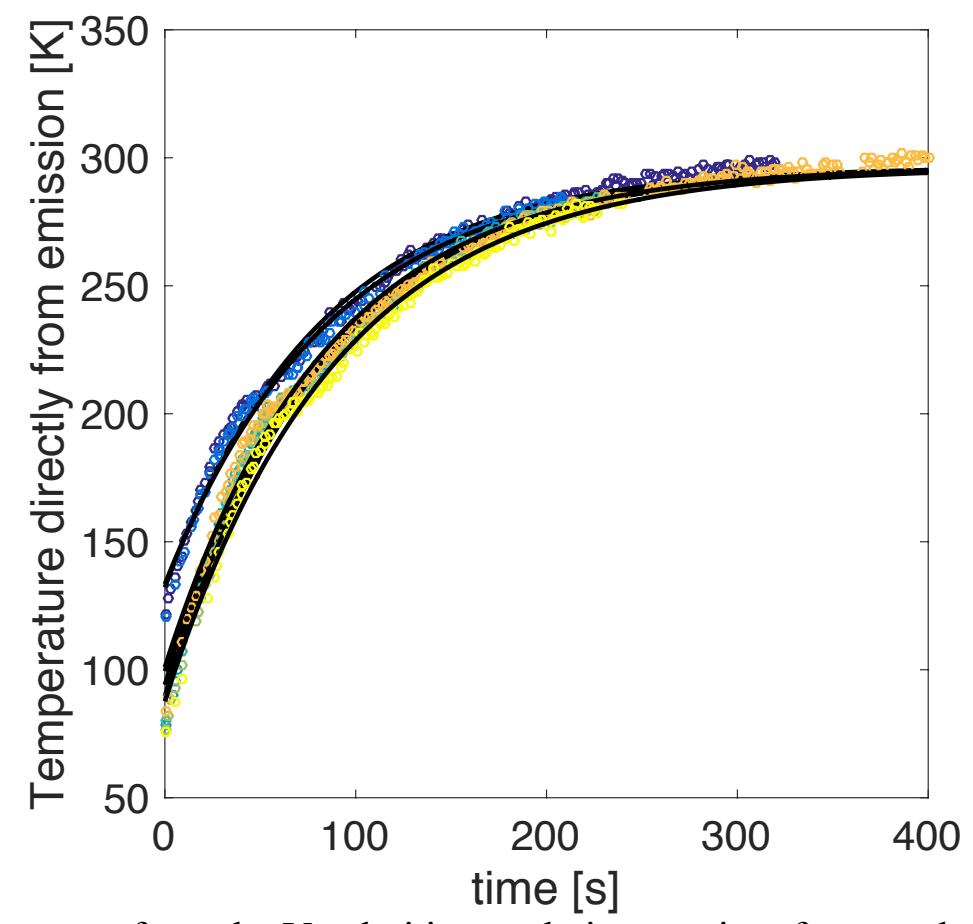

Figure S5: Temperature from the Varshni interpolation vs. time for sample ODPA36, The black lines show the Newton's law of cooling fit used to determine the final temperature. 
In order to calibrate the temperature, we first use a simple emission calibration, as was previously performed. ${ }^{\mathrm{S} 1 \mathrm{~S} 2}$ The Varshni relation, shown below empirically describes the change in semiconductor bandgap with temperature:

$$
E_{G}(T)=E_{G}(0)-\frac{\alpha T^{2}}{T+\beta}
$$

where the parameters are $E_{G}(0)=2.176 \mathrm{eV}, \alpha=0.29 \mathrm{meV} / \mathrm{K}, \beta=92.44 \mathrm{~K}$, similar to that of bulk CdSe, except for the $E_{G}(0)$, which is blue-shifted due to quantum confinement. Figure S3 shows the calibration curve generated via a temperature-dependent photoluminescence measurement performed in a cryostat. The resultant temperature-dependent PLQY calibration is shown in Figure S3. The calibration appears relatively accurate, however there is a non-monotonic behavior around the phase transition region that is clearly present in sample ODPA84. This behavior is clearly not physical, and can be explained by non-monotonic behavior in the temperature-dependent emission peak around the phase transition temperature (shown in the region around $200 \mathrm{~K}$ ). This is shown in Figure S5, in which the temperature from emission calibration is shown against time for sample ODPA36. Around the observed phase transition temperature there is a slight kink in the temperature calibration. This behavior could potentially be due to a size distribution present in the sample. If smaller dots disorder at lower temperatures, there will be a slight blue shift as temperature increases around the phase transition point. This would be most significant in samples that have a sharp phase transition, as is evident in Figure S3.

It is possible to improve the temperature calibration by adding another constraint: simply, that over time, the temperature increases as the sample warms up. This can be modeled using a fit with Newton's law of cooling:

$$
T(t)=T_{\text {room }}+\left(T_{\text {initial }}-T_{\text {room }}\right) e^{-k t}
$$

We use a global fit to the room temperature over each sample run to extract the appropriate parameters. The time constant $k$ is allowed to vary as it is possible slight changes in orientation could change the convection and thus the rate of heating and the time constant $T_{\text {initial }}$ is allowed to vary as the time between the sample being flash-frozen and the beginning of data collection is variable. The fits are shown in Figure S5. The result of this additional calibration step is to slightly increase the apparent width of the phase transition. This offsets the error from the calibration to a slight blue shift with increasing temperature that is present in the emission-only 
calibration method. Furthermore, it is clear that this fitting method resolves the unphysical nonmonotonic behavior that was observed in sample ODPA84. There is the potential for error that occurs while using this additional fitting. The $95 \%$ confidence intervals for the temperature calibration due to the Newton's law of cooling fit are shown in Figure S6. Above approximately $150 \mathrm{~K}$, the error due to the calibration is less than $2 \mathrm{~K}$. The higher error for temperature under $150 \mathrm{~K}$ is due partially to the fewer data points collected at lower temperatures, and the reliance of those points to provide the initial temperature. However for all samples the error does not exceed $5.5 \mathrm{~K}$, indicating a reasonable calibration.

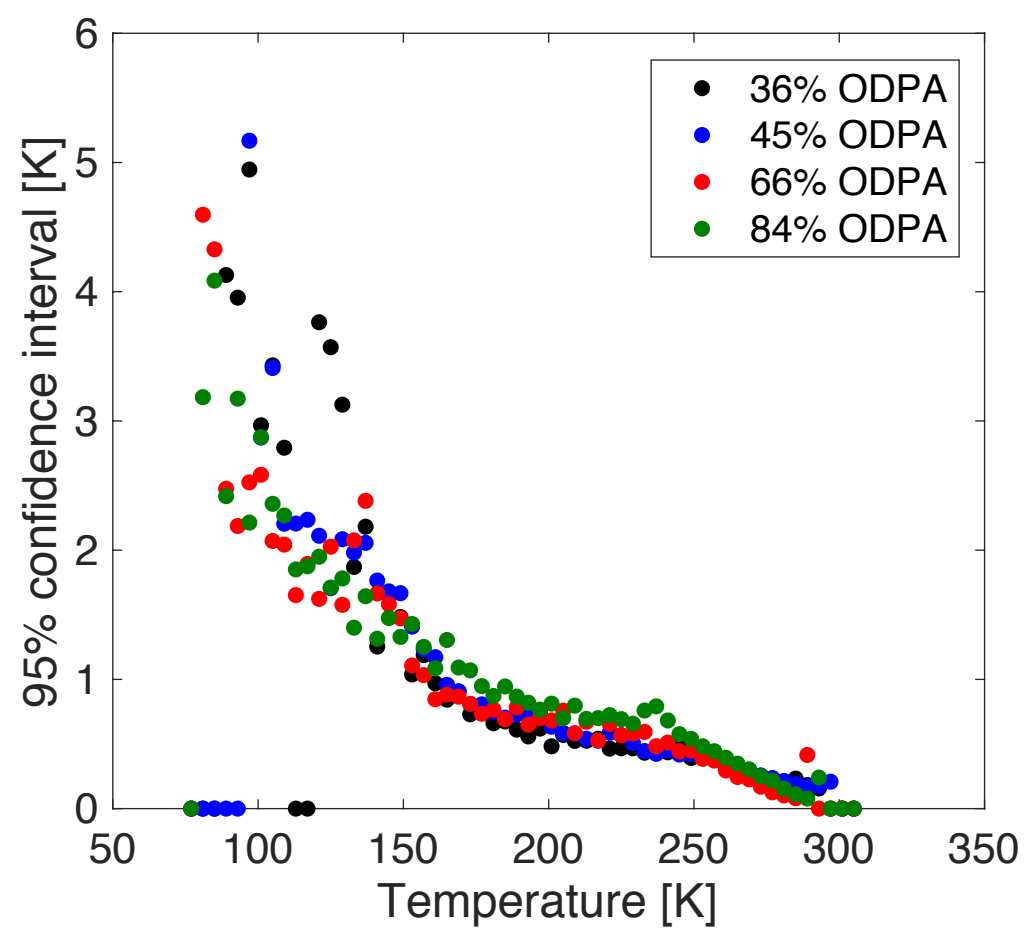

Figure S6: 95\% confidence interval for Newton's law of cooling fit., after binning data. The $0 \mathrm{~K}$ error points correspond to bins for which there are no data. 
Analyzing nonradiative and radiative rates vs. $\mathrm{T}$

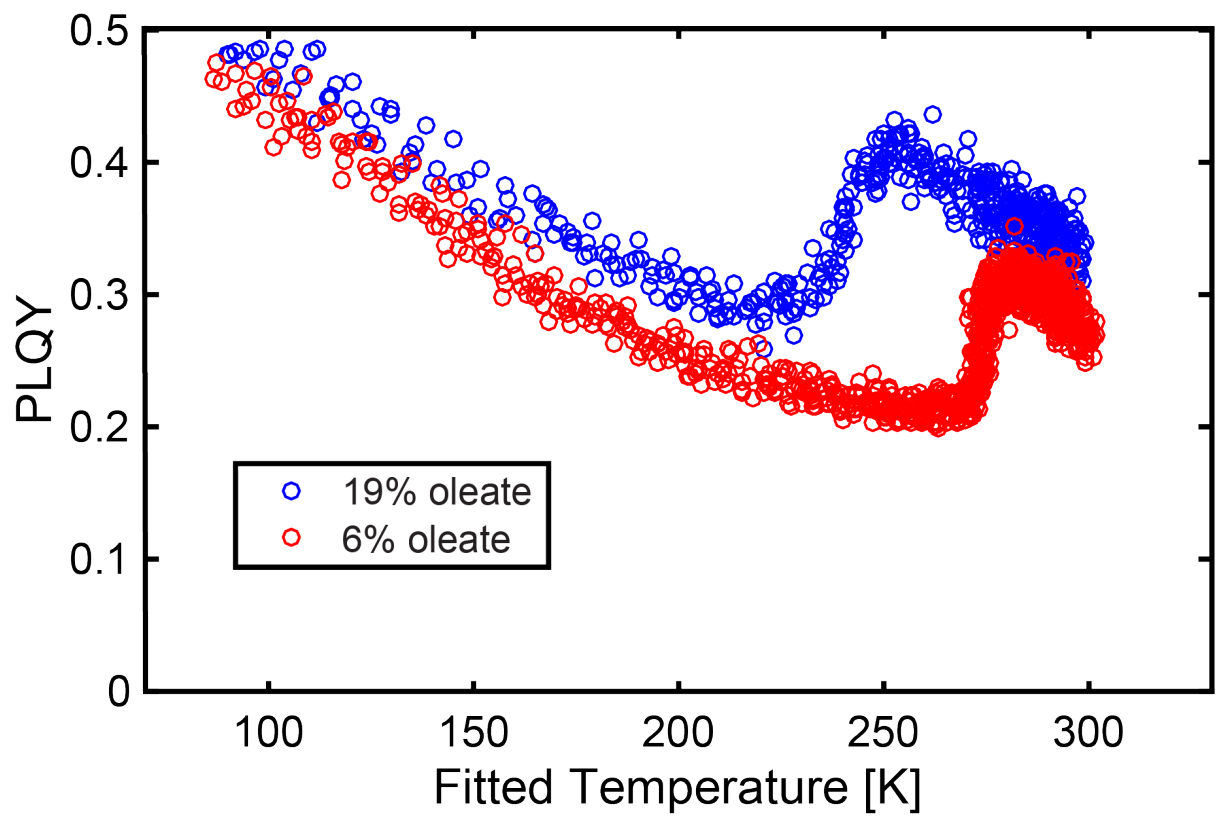

Figure S7: PLQY as a function of temperature (using only the emission calibration) for two different ligand compositions on another QD sample 

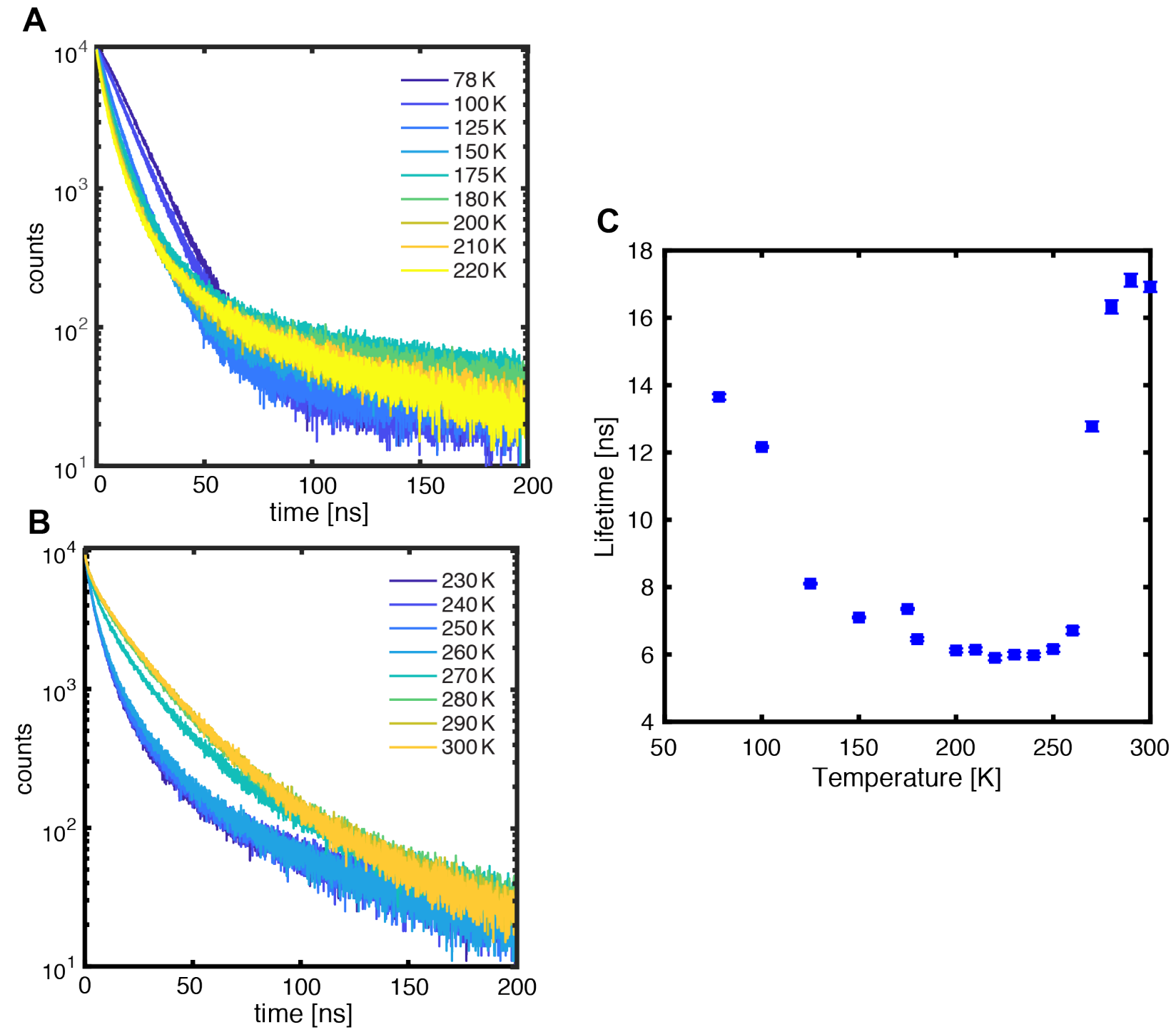

Figure S8: Temperature-dependent lifetime of the 6\% oleate sample shown in Figure S6. (A-B) shows a selection of the raw lifetime data, and $\mathrm{C}$ ) shows the resultant monoexponential fits to the full data set. 


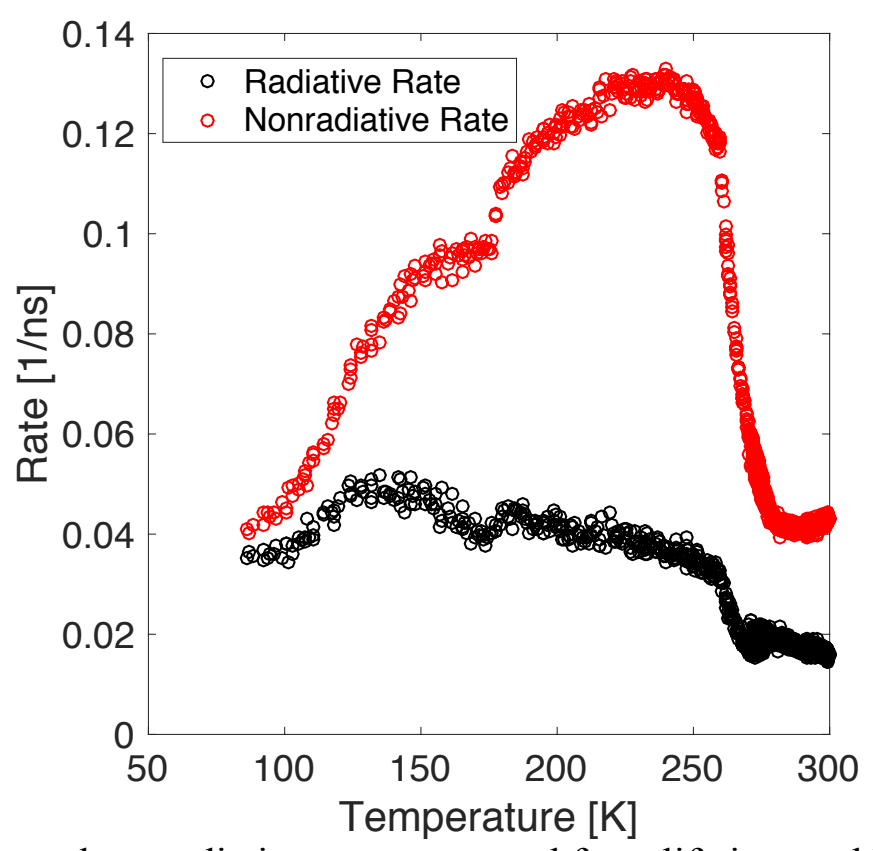

Figure S9: Radiative and nonradiative rates extracted from lifetime and PLQY data for the $6 \%$ oleate sample. The small decrease in the radiative rate around $270 \mathrm{~K}$ with increasing temperature is likely due to small errors in interpolation of the temperature-dependent lifetime.

To compare the degree to which measuring the PLQY and measuring the lifetime agree, both measurements were performed on a different QD sample. As shown in Figures S7 and S8, the change in PLQY and lifetime with temperature agree well. This is further highlighted in Figure S9, which shows the change in radiative and nonradiative rate with temperature. To achieve this result, both the lifetime and PLQY data were used. This highlights two features. First, as expected, the major parameter that changes during the phase transition is the nonradiative rate. Secondly, the agreement between the fast PLQY measurement and the slow (performed in a cryostat) lifetime measurement confirms that the different rates at which the experiment is performed, over minutes for the PLQY measurements and hours for the lifetime measurements, suggest that the kinetics of the phase transition is not driving the observed behavior. It further supports that the manner by which temperature is calibrated in the PLQY measurements is not creating an artificial sharpness to the observed change in PLQY and FWHM with temperature. 


\section{Variable temperature IR data}

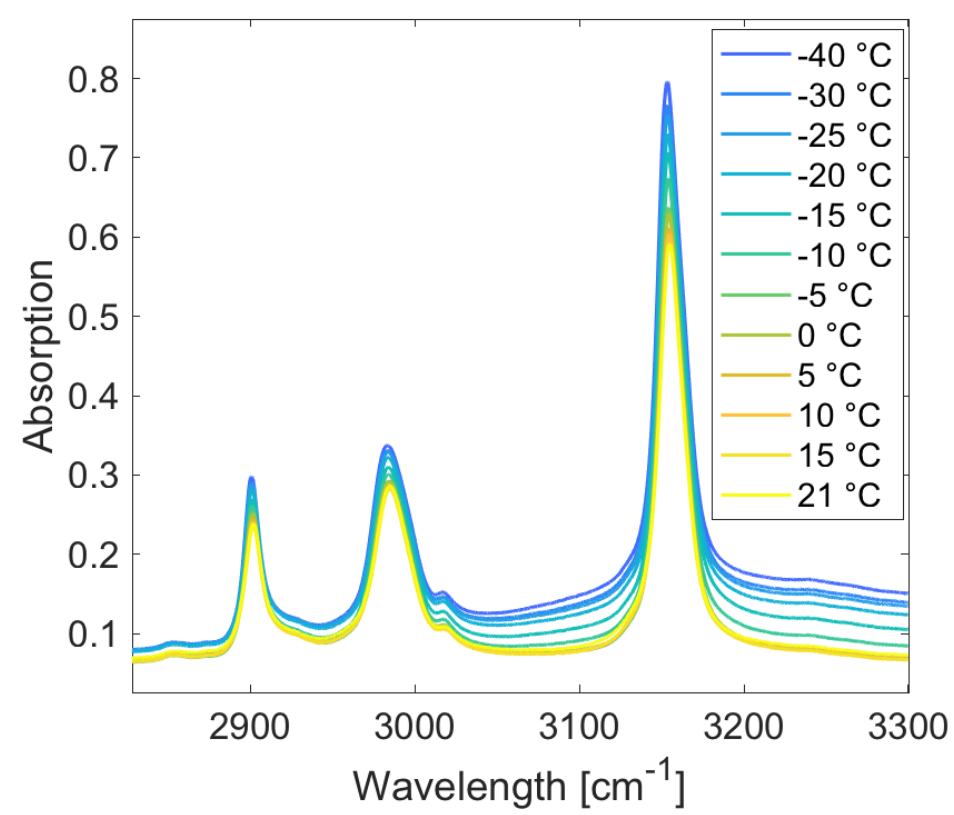

Figure S10: Temperature-dependent $\mathrm{CDCl}_{3}$ IR baseline spectra
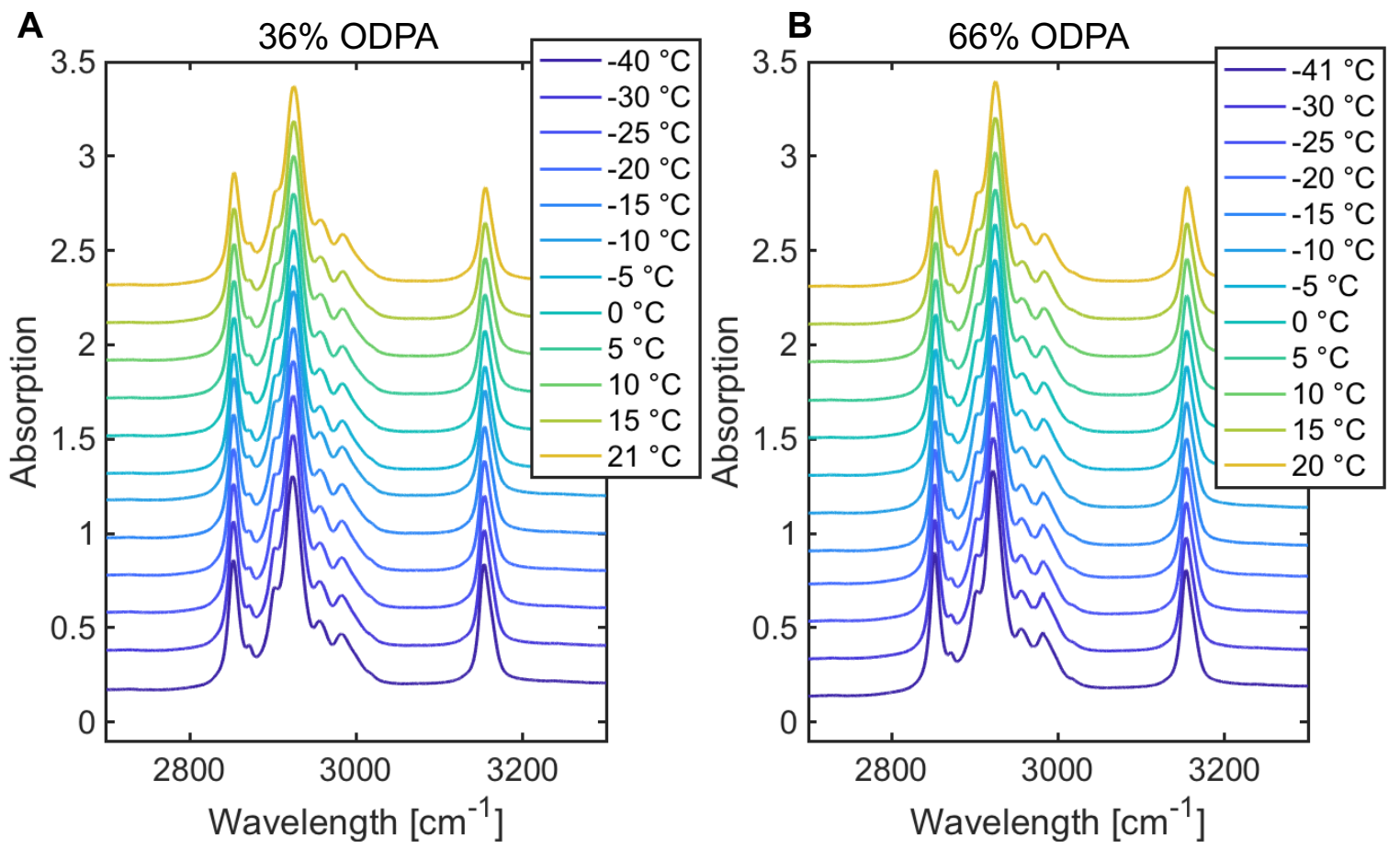

Figure S11: Temperature-dependent IR spectra for A) sample ODPA36 and B) sample ODPA66, shown without any solvent or baseline subtraction. 

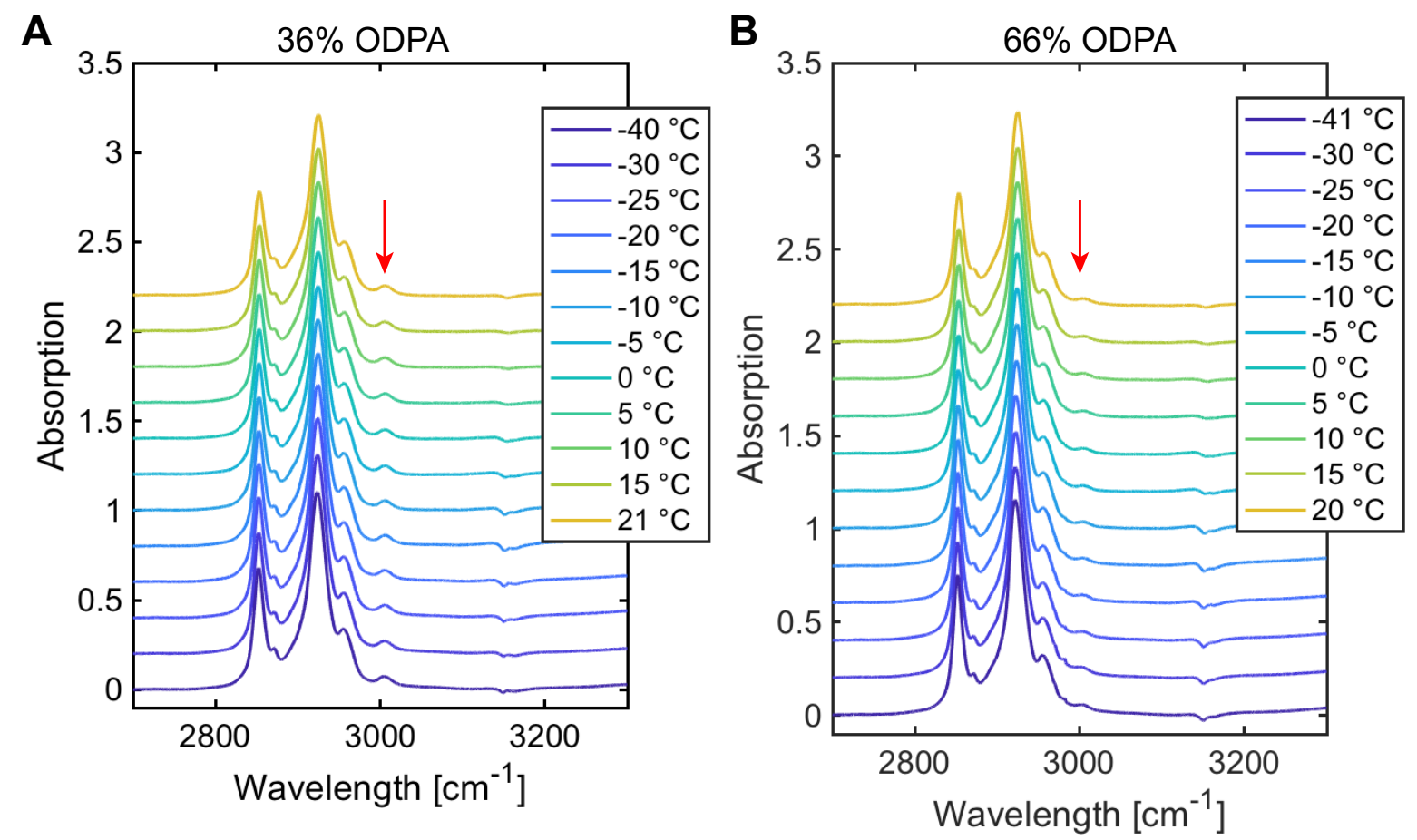

Figure S12: Temperature-dependent IR spectra for A) sample ODPA36 and B) sample ODPA66. The data has been subtracted from the $\mathrm{CDCl}_{3}$ background and baseline corrected. The vinyl $\mathrm{C}-\mathrm{H}$ stretching peak is emphasized with a red arrow.

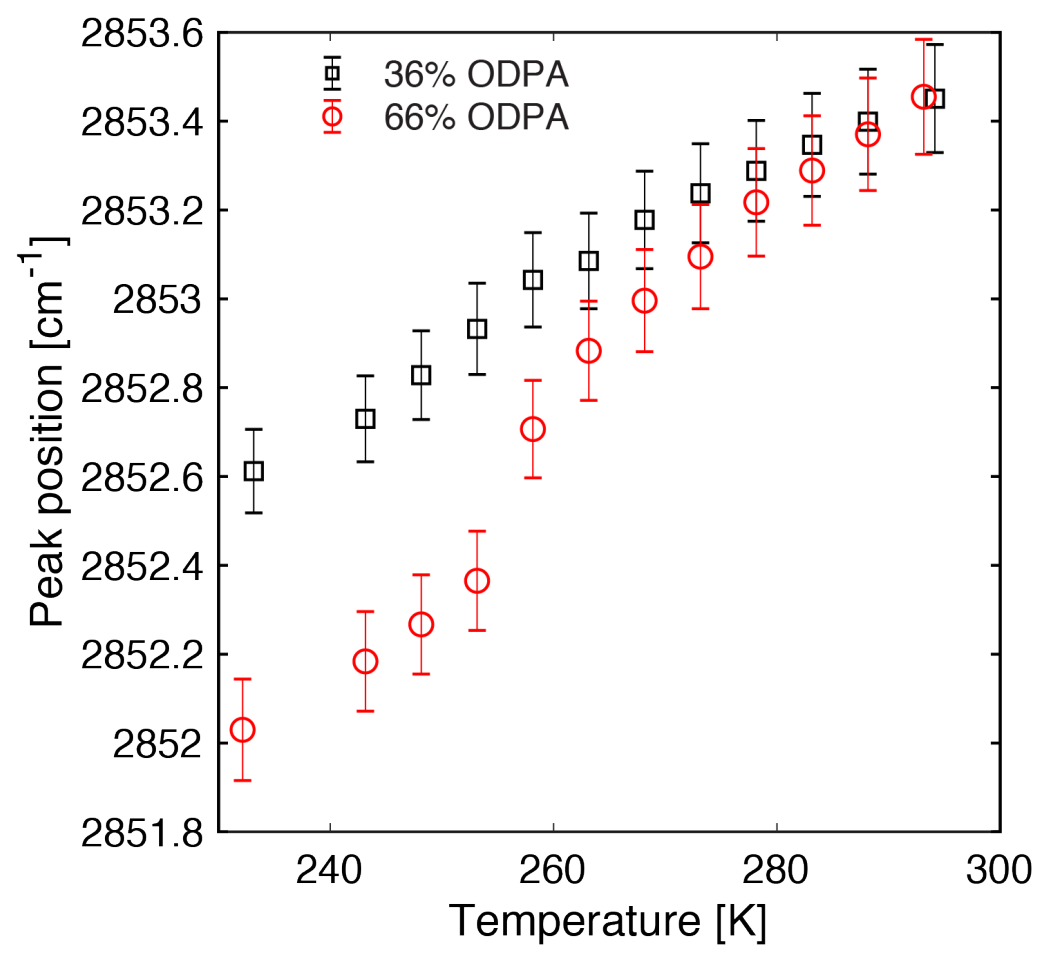

Figure S13: Symmetric stretching peak position 
$\mathrm{CDCl}_{3}$ was chosen as the VT-IR solvent because the $\mathrm{CDCl}_{3}$ IR spectra, shown in Figure $\mathrm{S} 10$, does not significantly overlap with the relevant $\mathrm{C}-\mathrm{H}$ stretches in the aliphatic chains, as highlighted in Figure S11. In addition, QD solubility in $\mathrm{CDCl}_{3}$ is high, which allowed for high QD loading, and improved IR signal from the methylene chains. Figure S12 shows the entire C$\mathrm{H}$ stretching region for sample ODPA36 and ODPA66, with the vinyl stretch highlighted with a red arrow. Despite overlapping with the $\mathrm{CDCl}_{3}$ baseline, we can still observe the change in vinyl stretch intensity from sample ODPA36 to sample ODPA66. In addition, Figure S12 indicates a successful subtraction of the $\mathrm{CDCl}_{3}$ solvent peaks, with only small derivative features present in Figure S12.

In Figure 4 of the main text, we show the asymmetric methylene stretch change with temperature. Shown in Figure S13 is the change with temperature for the symmetric stretch. We see a similar trend, in which sample ODPA66 shows a sharp increase in the symmetric stretching peak position around the observed phase transition temperature, which is not observed for sample ODPA36. However, the magnitude of change in the symmetric stretch is rather smaller than that for the asymmetric stretch. This is somewhat surprising, as previous reports have asserted that the symmetric stretch is more sensitive to the degree of disorder of the aliphatic chains than the asymmetric stretch. ${ }^{\mathrm{S} 3, \mathrm{~S} 4}$ However, the same general conclusions can be drawn from the both the symmetric and asymmetric stretch: that the phase transition involves an increase in the number of gauche defects present in the aliphatic chains, and that in the more ordered state, there still is present a rather significant amount of disorder. 


\section{SFG data and analysis}
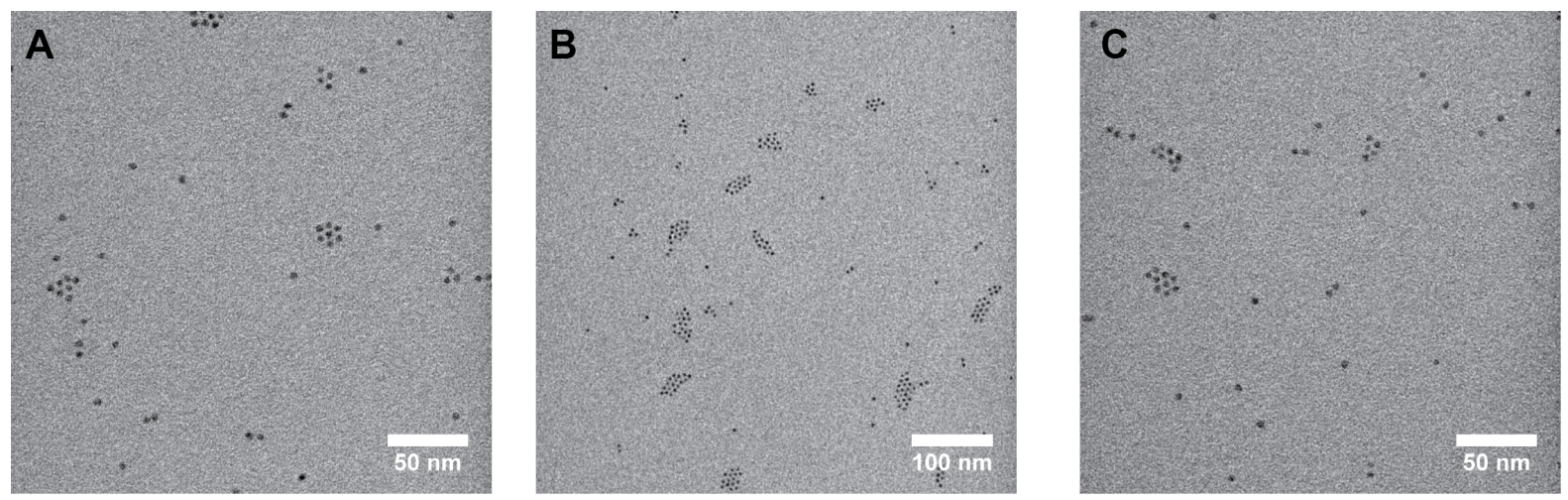

Figure S14: TEM micrographs of CdSe QD film density after spin-coating (using the same conditions as described in the main text) taken from the A) Center, B) halfway to the edge, and C) edge of the SFG substrate. 


\begin{tabular}{|c|c|c|c|c|c|c|c|}
\hline & & & ODPA 36\% & & & ODPA 84\% & \\
\hline & & $30 \mathrm{C}$ & $20 \mathrm{C}$ & $10 \mathrm{C}$ & $30 \mathrm{C}$ & $20 \mathrm{C}$ & $10 \mathrm{C}$ \\
\hline \multirow[t]{3}{*}{$\mathrm{CH} 2$ stretch } & peak & 2810 & 2804 & 2817 & 2802 & 2795 & 2808 \\
\hline & amplitude & 0.8161 & 1.146 & 0.5848 & 1.324 & 2.49 & 1.303 \\
\hline & width & 21 & 20.5 & 19 & 25 & 30 & 16 \\
\hline & & & & & & & \\
\hline \multirow[t]{3}{*}{ sym-CH2 } & peak & 2849 & 2846 & 2850 & 2845 & 2860 & 2849 \\
\hline & amplitude & 1.125 & 1.589 & 1.089 & 1.25 & 2.274 & 2.348 \\
\hline & width & 10.5 & 11 & 9 & 18 & 20 & 16 \\
\hline & & & & & & & \\
\hline \multirow[t]{3}{*}{ sym-CH3 } & peak & 2884 & 2890 & 2883.1 & 2878 & 2887 & 2873 \\
\hline & amplitude & 0.2 & 0.1804 & 0.1558 & 0.1189 & 0.2919 & 1.329 \\
\hline & width & 8 & 8.5 & 8 & 10 & 11 & 13 \\
\hline & & & & & & & \\
\hline \multirow[t]{3}{*}{ asym-CH2 } & peak & 2916 & 2914 & 2909 & 2915 & 2920 & 2915 \\
\hline & amplitude & 0.5164 & 0.7711 & 0.962 & 1.047 & 0.5531 & 2.344 \\
\hline & width & 10 & 13.8 & 16 & 14 & 9 & 14 \\
\hline & & & & & & & \\
\hline \multirow[t]{3}{*}{$\mathrm{FR}-\mathrm{CH} 2$} & peak & 2929 & 2931 & 2928 & 2935 & 2930 & 2935 \\
\hline & amplitude & 0.34659 & 0.3087 & 0.3181 & 0.1343 & 0.8077 & 1.955 \\
\hline & width & 12 & 8 & 8 & 15 & 13 & 17 \\
\hline & & & & & & & \\
\hline \multirow[t]{3}{*}{$\mathrm{FR}-\mathrm{CH} 3$} & peak & 2948 & 2955 & 2945 & 2954 & 2960 & 2950 \\
\hline & amplitude & 0.144 & 0.1 & -0.231 & 1.839 & 3.512 & 3.838 \\
\hline & width & 10 & 20 & 10 & 1.839 & 19 & 15 \\
\hline & & & & & & & \\
\hline \multirow[t]{3}{*}{ asym-CH3 } & peak & 2977 & 2970 & 2978 & 2970 & 2968 & 2970 \\
\hline & amplitude & 0.2851 & 1.2854 & 1.392 & 0.1 & 0.1389 & 0.1 \\
\hline & width & 12.3 & 13.5 & 20 & 20 & 10 & 20 \\
\hline \multirow{4}{*}{$\mathrm{C}=\mathrm{C}-\mathrm{H}$ stretc } & & & & & & & \\
\hline & peak & 3018 & 3016 & 3011 & 3022 & 3019 & 3018 \\
\hline & amplitude & 0.5666 & 0.7119 & 0.7599 & 0.6667 & 2.984 & 0.8977 \\
\hline & width & 15 & 15 & 12 & 21 & 35 & 12 \\
\hline
\end{tabular}

Table S1: SFG peak fitting parameters 


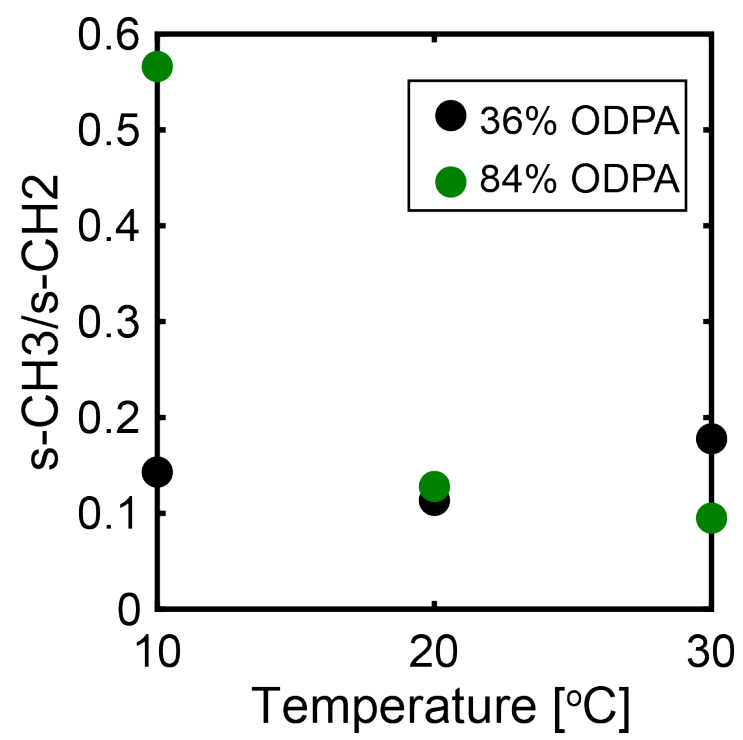

Figure S15: Ratio between symmetric methyl and methylene stretches for SFG samples

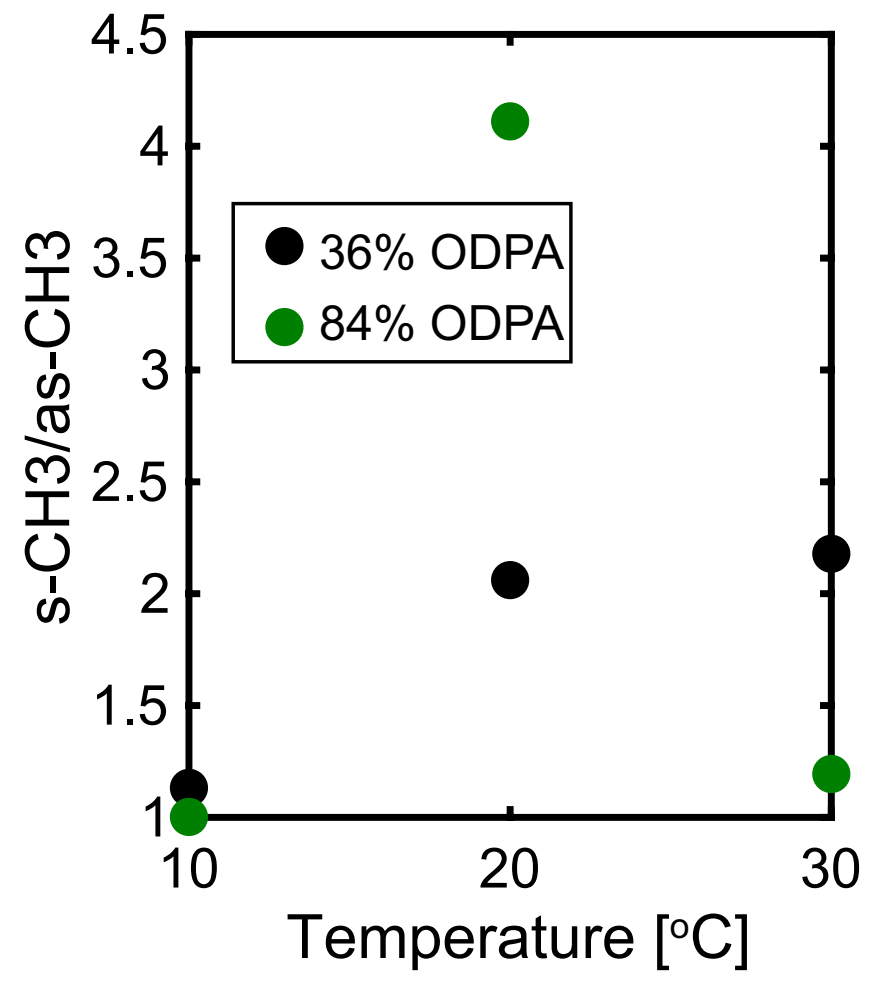

Figure S16: Ratio between symmetric and asymmetric methylene stretches for SFG samples 


\section{Concentration- and binding group-dependence of PL data}

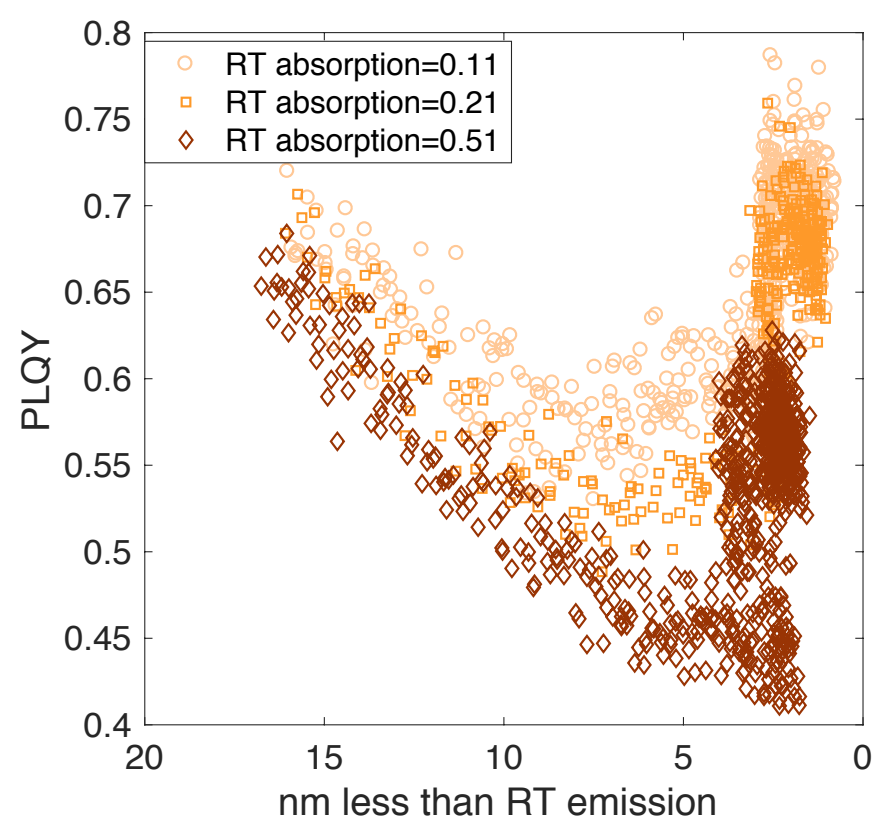

Figure S16: Concentration dependence of temperature-dependent PLQY of sample ODPA84. Due to the increasing non-monotonic character of the curves, they are plotted against the nm less than RT emission, a proxy for temperature.
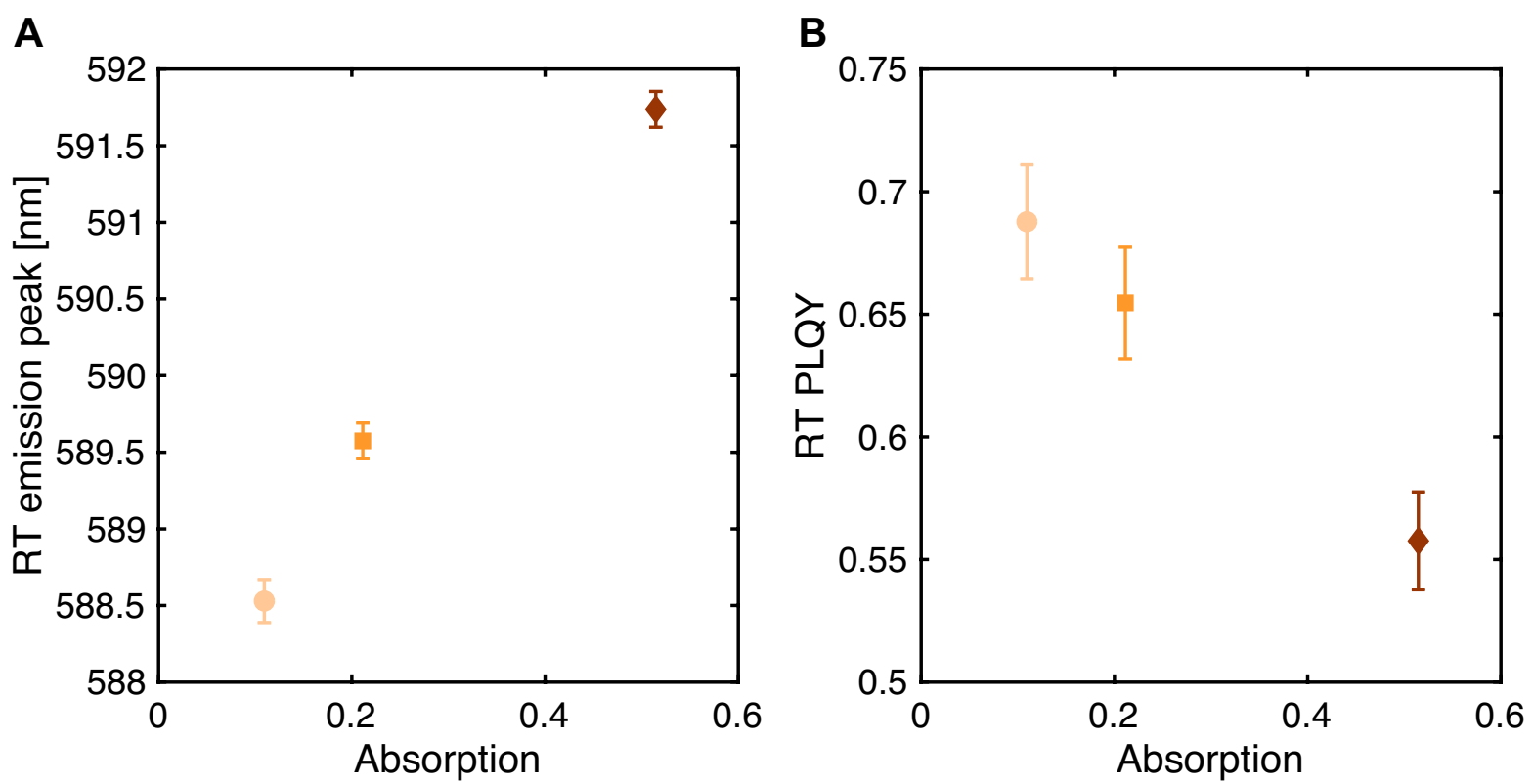

Figure S17: Dependence of the room temperature emission peak (a) and PLQY (b) on sample ODPA84 
One possibility we set out to exclude was that the change in PLQY at the phase transition is due to a change in interparticle interactions as opposed to a change on a single particle level. Namely, it is possible that the phase transition is not driven by a change in the structure of the aliphatic chains, but by an aggregation of the QDs themselves. It is likely that this aggregation behavior would also depend on the ligand composition, so it is important to address. To address this behavior, we first performed concentration-dependent on sample ODPA84, as shown in Figure S17. We note the emission comparison is slightly different as compared to previous samples, as an increase in concentration results in a redshift of the emission peak due to absorption and reemission, which explains the observed decrease in PLQY for the higher concentration sample, as shown in Figure S18. Figure S17 indicates that there is not a significant change in the phase transition temperature for three different concentrations. This would suggest that aggregation of particles is not driving the observed phase transition. Additionally, we do note that the phase transition temperature observed in Figure S17 is different than that of Figure 3 and Figures S3-4. This is due to the fact that S17 was measured significantly after the former measurements. It is possible that over time the $\mathrm{CdX}_{2}$ ( $\mathrm{X}=\mathrm{ODPA}$ or $\mathrm{OA}$ ) complexes desorb from the $\mathrm{QD}$ surface, thus changing the ligand composition or density, affecting the phase transition temperature.

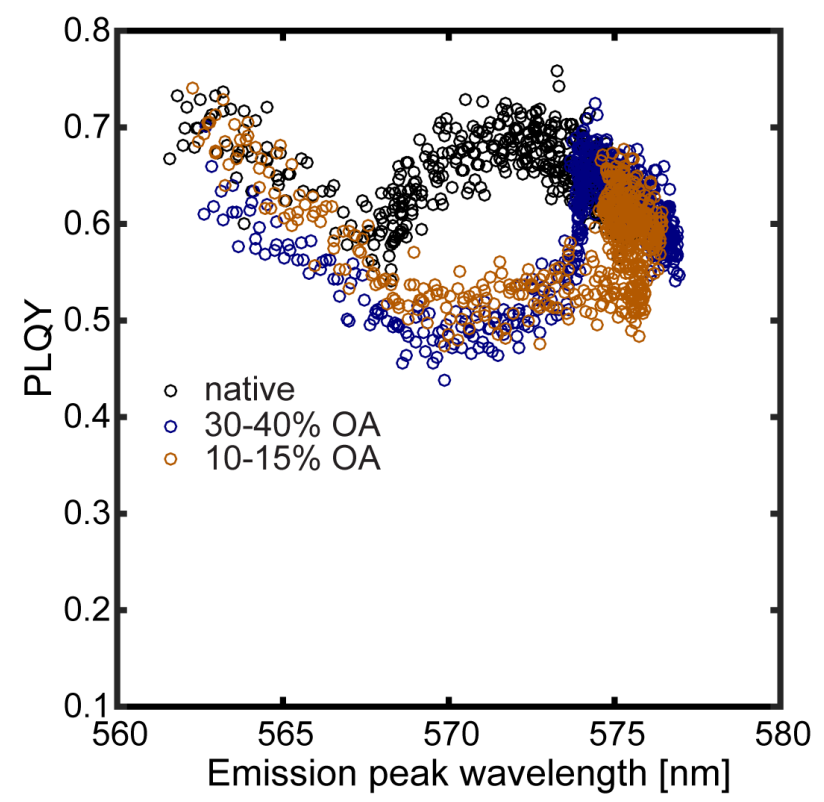

Figure S18: Temperature-dependent PLQY for a sample exchanged with stearic acid. 
In addition, ligand exchanges from $\mathrm{OA}$ to stearic acid were performed on a third sample of QDs. The temperature-dependent PLQY is shown in Figure S19 as a function of emission peak wavelength, which correlates with temperature. It clearly shows that as the amount of OA is decreased (amount of stearic acid is increased), the observed phase transition shifts to higher temperatures and narrows, as shown in Figure 3 of the main text and Figure S7. This shows that the change in PLQY is not due to a change in the amount of ligand debinding. Rather, the chain type plays a larger role in determining the phase behavior. 


\section{Size scaling of the phase transition}
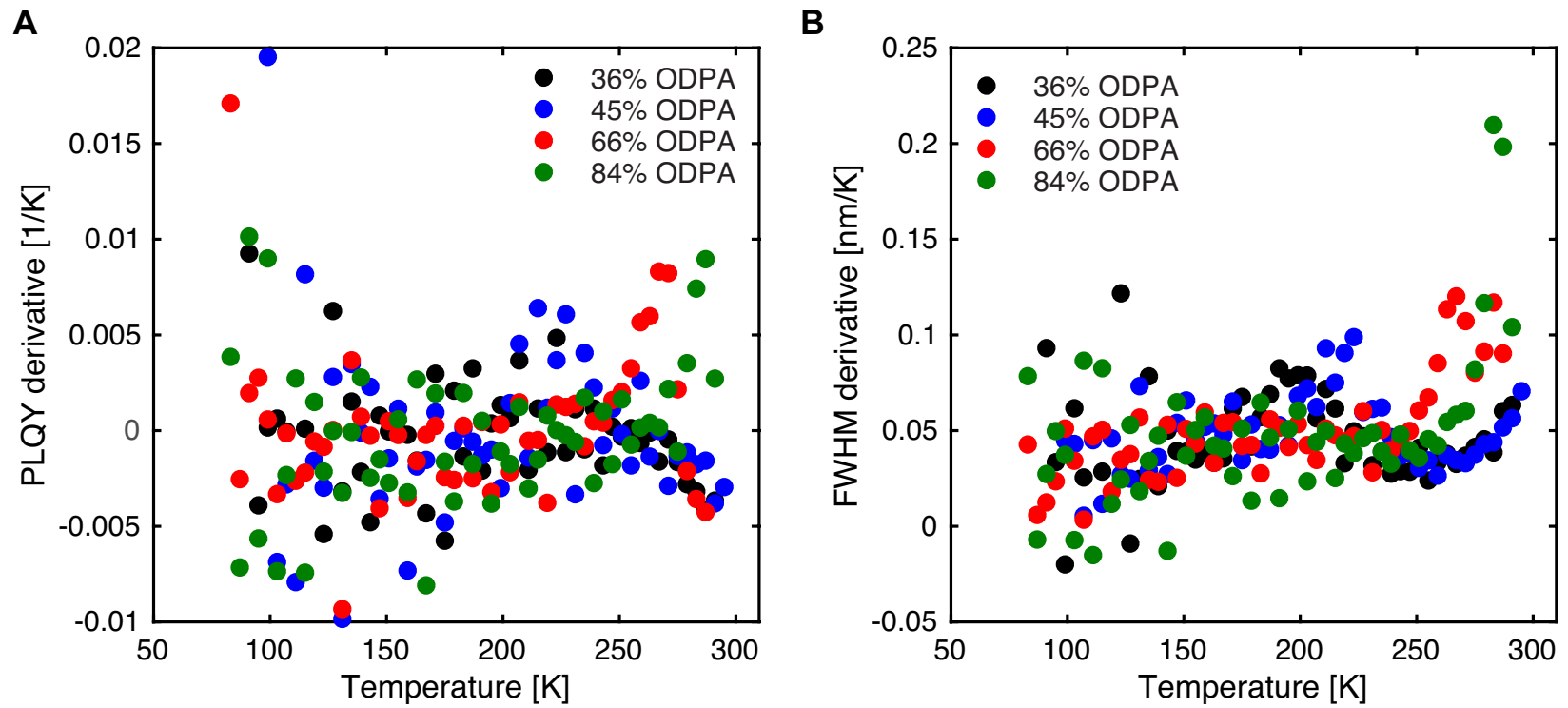

Figure S19: Numerical derivative of the binned PLQY (a) and FWHM (b) data shown in Figure 3 of the main text. The FWHM analysis was performed on FWHM measured in nm.

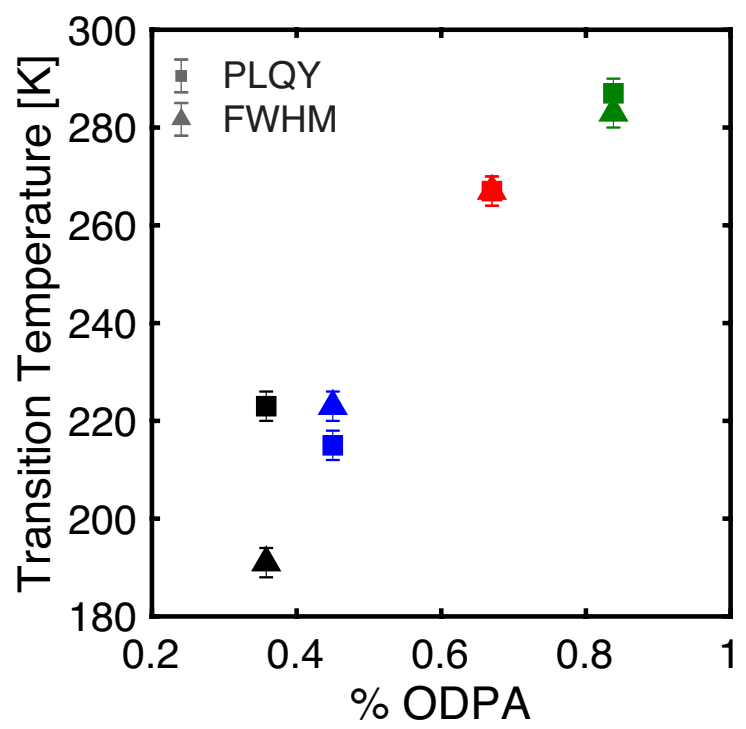

Figure S20: The phase transition temperature extracted using the maximal numerical derivative, as shown in Figure S20

The raw temperature-dependent photoluminescence data can be binned to improve the clarity of the PLQY data, and has been shown in Figure 3 of the main text. In order to determine the phase transition temperature, it is possible to determine the maximal derivative using a numerical approach, where the slope is calculated in between each bin. The numerical derivative of the 
binned data is shown in Figure S20. This data has significant noise, which indicates the pitfalls of this analysis method. Additionally, the (additive) error bars for this analysis are extremely large, and are not shown in Figure S20. Despite the noise present in the data, it is possible to observe the phase transition regions. Particularly for the FWHM data a peak is apparent consistent with the phase transition temperature for all samples. For the PLQY data, for all samples except for the $36 \%$ ODPA sample, it is possible to observe a peak corresponding to the phase transition. The relevant maximal derivatives are shown in Figure S21. They match relatively well to the phase transition temperatures extracted using a fit to the Cauchy cumulant, as shown in Figure 6 of the main text. The main error arises from the 36\% ODPA sample PLQY, where there does appear to be a broad transition region between $150-220 \mathrm{~K}$ or so, but the derivatives are all quite similar, and therefore the maximal derivative is more likely to be inaccurate. For the above reasons, in the main text we implemented a fit to the Cauchy distribution integral. 
Cauchy distribution integrals to fit phase transition

A

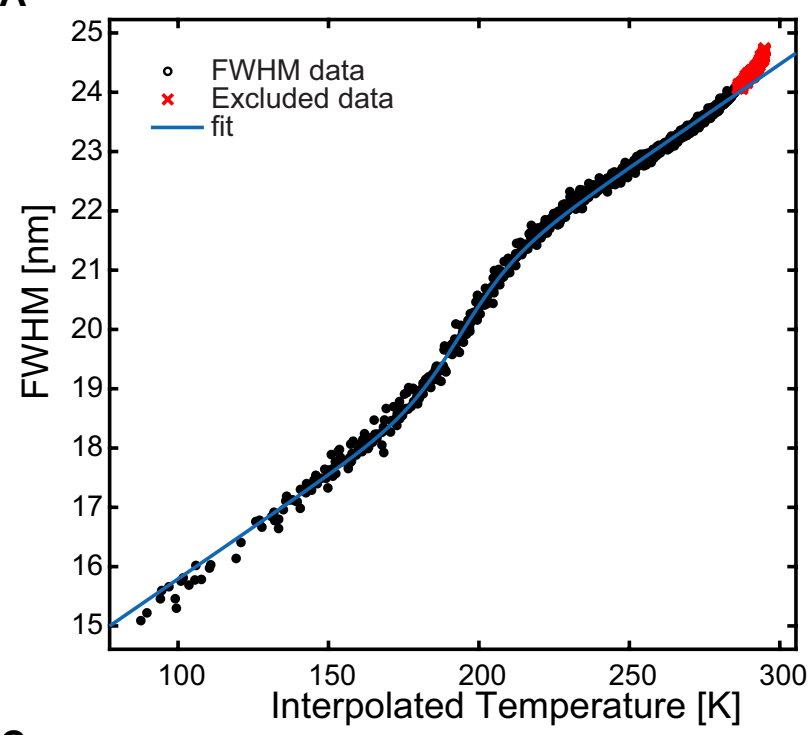

C

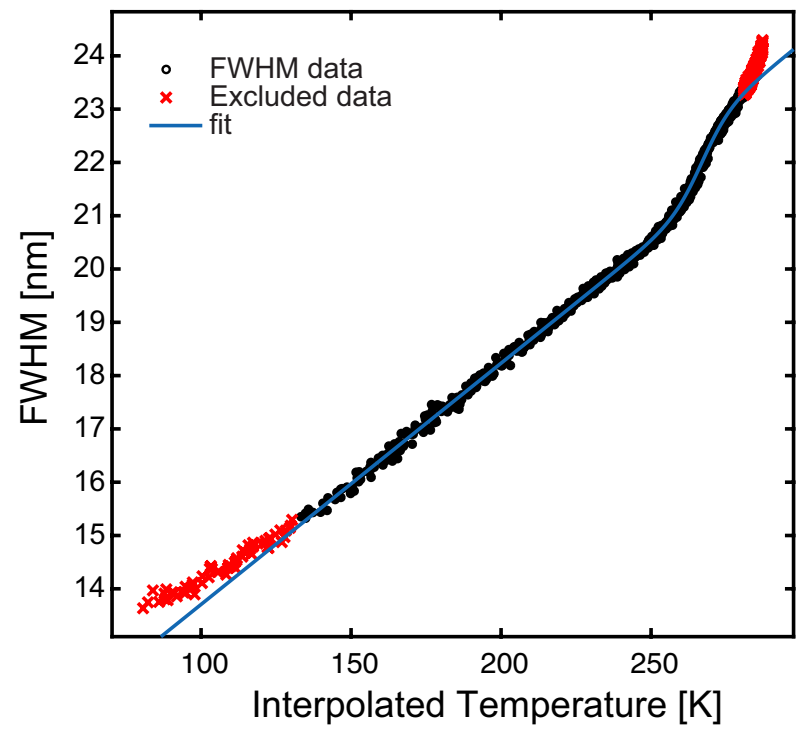

B
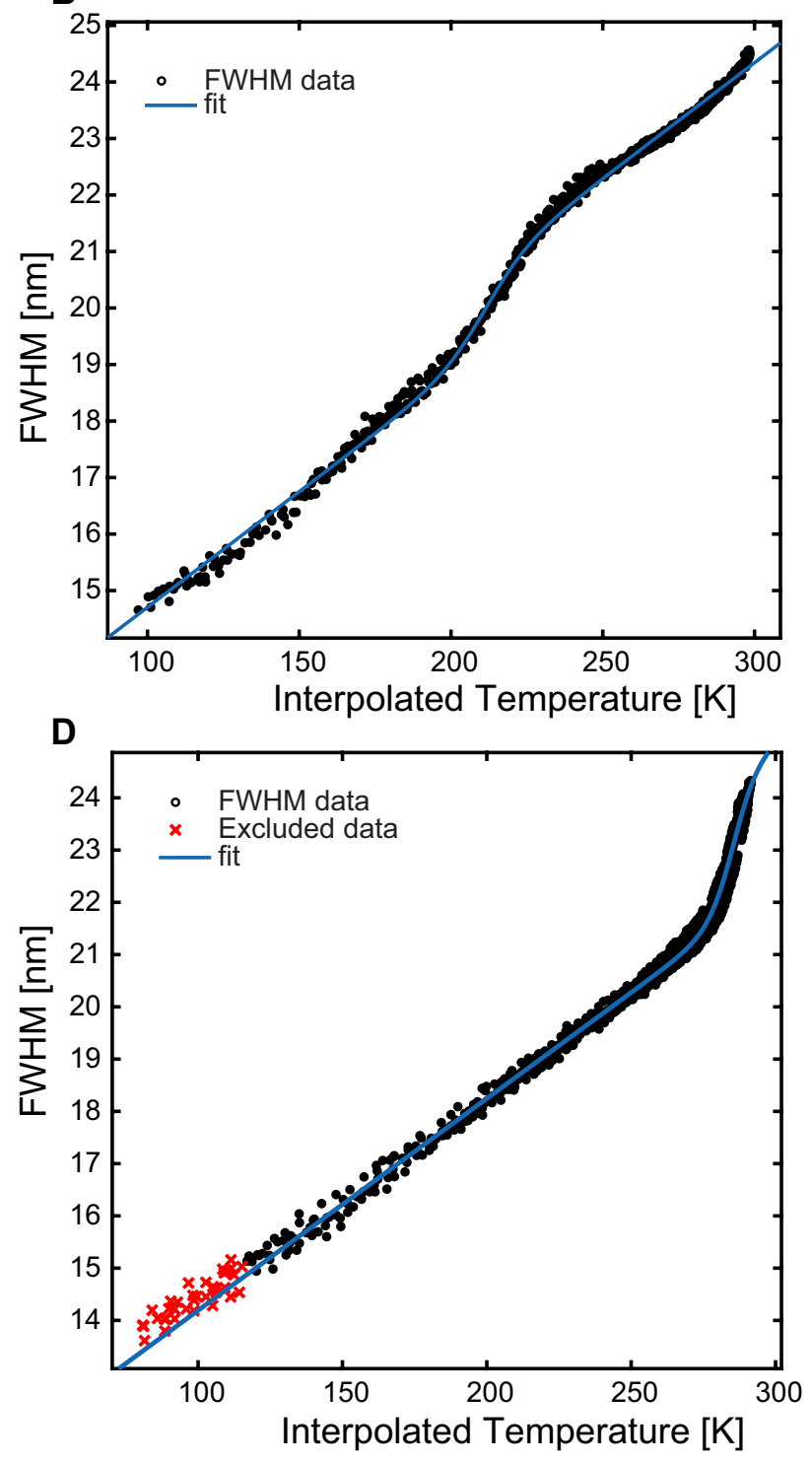

Figure S21: Cauchy distribution fits for the FWHM for samples A) ODPA36, B) ODPA45, C) ODPA66, D) ODPA84. 


\begin{tabular}{|l|l|l|l|l|l|}
\hline Sample & $\mathrm{O}_{\mathrm{o}}[\mathrm{nm}]$ & $\mathrm{b}[\mathrm{nm} / \mathrm{K}]$ & $\mathrm{c}[\mathrm{nm}]$ & $\mathrm{T}_{\mathrm{PT}}[\mathrm{K}]$ & $\Delta \mathrm{T}[\mathrm{K}]$ \\
\hline ODPA36 & $13.16(0.11)$ & $.03488(0.00053)$ & $.8517(.0393)$ & $193.9(0.6)$ & $18.66(1.46)$ \\
\hline ODPA45 & $11.34(0.13)$ & $.04092(0.00058)$ & $.7262(.0376)$ & $211.4(0.7)$ & $14.65(1.73)$ \\
\hline ODPA66 & $\begin{array}{l}9.919 \\
(0.077)\end{array}$ & $\begin{array}{l}0.04526 \\
(0.00029)\end{array}$ & $.739(.024)$ & $267(0.4)$ & $9.769(.643)$ \\
\hline ODPA84 & $11.55(0.19)$ & $.04055(0.00046)$ & $1.414(.126)$ & $285.5(0.8)$ & $8.518(0.92)$ \\
\hline
\end{tabular}

Table S2: Fitting parameters for the FWHM
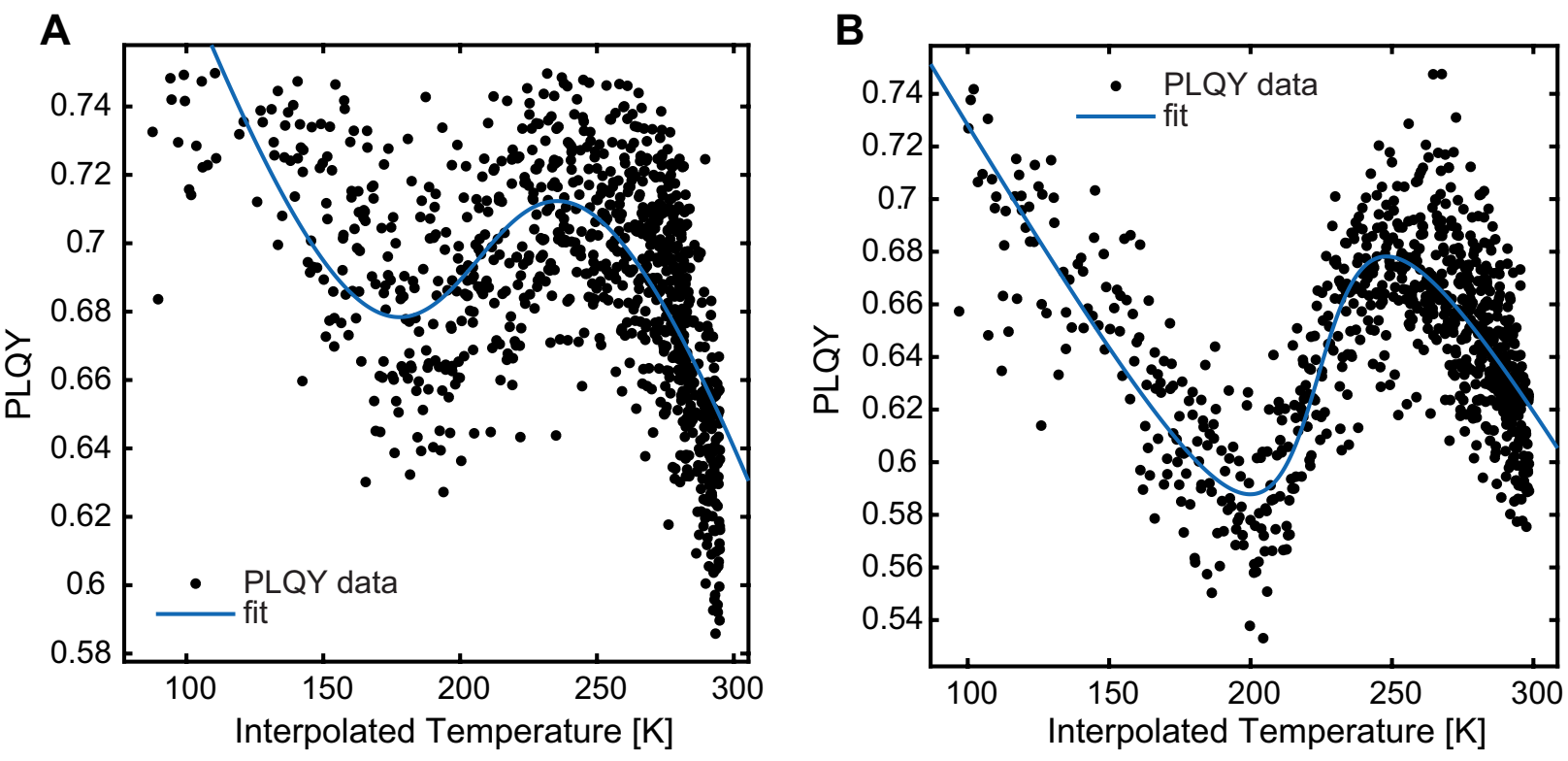

C

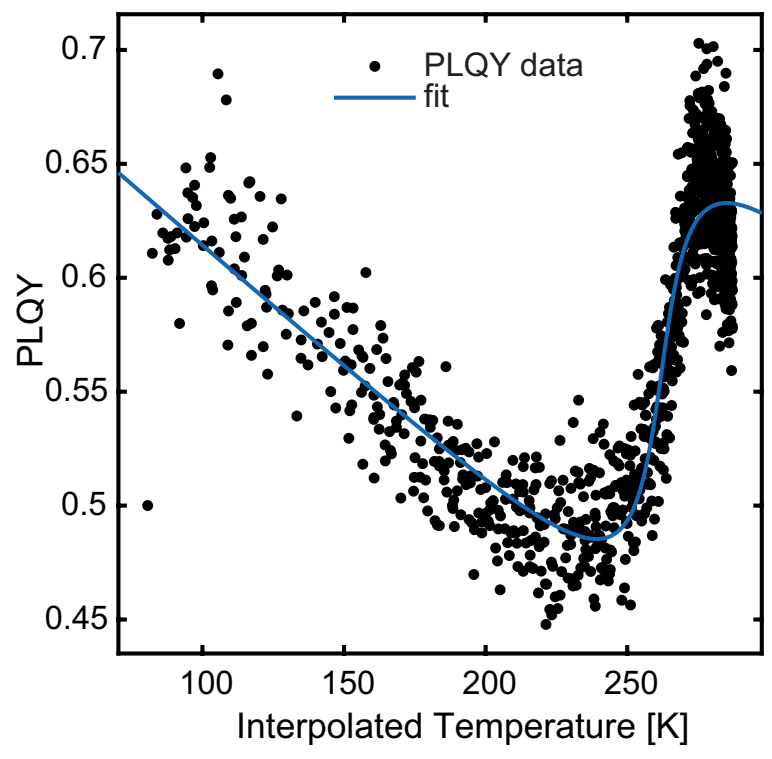

D

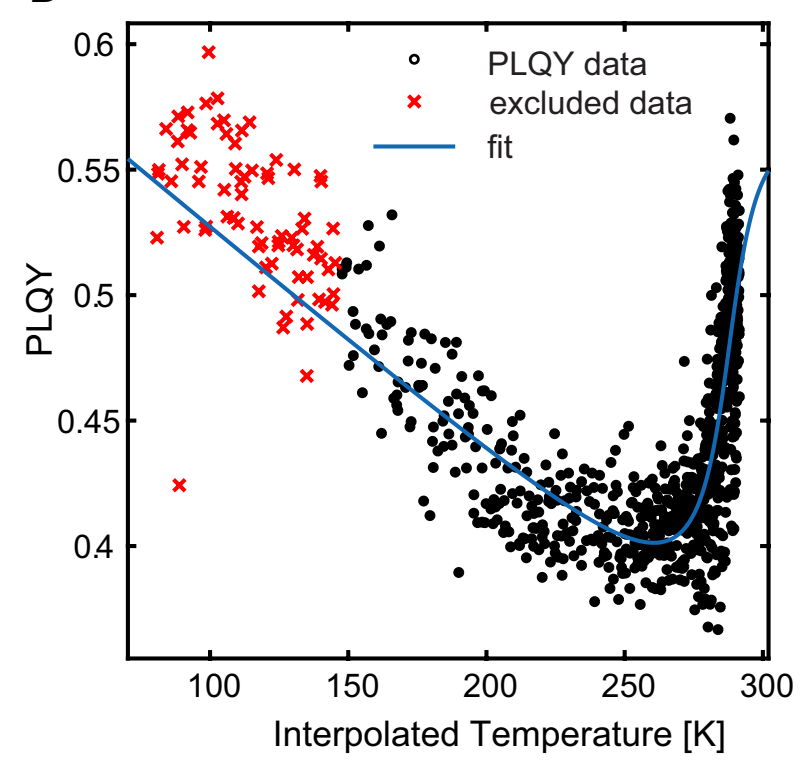


Figure S22: Cauchy distribution fits for the PLQY for samples A) ODPA36, B) ODPA45, C) ODPA66, D) ODPA84.

\begin{tabular}{|l|l|l|l|l|l|}
\hline Sample & $\mathrm{O}_{\mathrm{o}}$ & $\mathrm{b}[1 / \mathrm{K}]$ & $\mathrm{c}$ & $\mathrm{T}_{\mathrm{PT}}[\mathrm{K}]$ & $\Delta \mathrm{T}[\mathrm{K}]$ \\
\hline ODPA36 & $1.213(.167)$ & $.002505(8.17 \mathrm{e}-4)$ & $.1618(.0888)$ & $\begin{array}{l}206.7 \\
(2.3)\end{array}$ & $46.89(17.86)$ \\
\hline ODPA45 & $1.05(.05)$ & $.001863(2.24 \mathrm{e}-4)$ & $.09485(.01547)$ & $\begin{array}{l}224 \\
(1.3)\end{array}$ & $17.24(3.86)$ \\
\hline ODPA66 & $0.8455(.018)$ & $.001093(7.1 \mathrm{e}-5)$ & .08017 & $\begin{array}{l}262.1 \\
(.00486)\end{array}$ & $8.077(1.208)$ \\
& & & $.08341(.02605)$ & $\begin{array}{l}287.9 \\
(3.3)\end{array}$ & $9.226(2.361)$ \\
\hline ODPA84 & $.747(.062)$ & $9.28 \mathrm{e}-4(1.452 \mathrm{e}-$ & .08 & \\
\hline
\end{tabular}

Table S3: Fitting parameters for the PLQY

Fits for the Cauchy distribution integrals are shown in Figures S22 and S23 and Tables S2 and S3. To ensure accurate fitting of the distribution, points away from the phase transition region that show deviations from linearity are excluded in some of the fits. In particular, there is a curvature present near room temperature for the FWHM and thus were excluded from the fits. 


\section{Ising model of phase transition}

The Ising model assumes a two-state system consisting of spins of $+/-1$. The energy of the system is describing by the following equation:

$$
E=-H \sum_{i}^{N} i-J \sum_{\langle i j\rangle}^{\prime} i * j
$$

For the interacting aliphatic chains, we assume $\mathrm{i}=1$ for the ordered state, and $\mathrm{i}=-1$ for the disordered state. Assuming an energy difference $\epsilon=109.5 \mathrm{meV}$ associated with an isolated chain disordering, and a favorable interaction energy $\mathrm{W}$ only between the disordered chains, and an entropy of the disordered phase $S=14.6 k_{B}$, the phase transition can be mapped to the Ising model as follows:

$$
H(T)=\frac{\epsilon}{2}+W-\frac{S T}{2}
$$

and

$$
J=\frac{W}{4}
$$

The phase transition temperature for the $100 \%$ ODPA sample would occur at $\mathrm{H}(\mathrm{T})=0$, which is:

$$
T_{P T}=\frac{\epsilon+2 W}{S}
$$

The three parameters in this system are inter-related and likely different combinations of the three parameters can yield very similar results. Changing the $\epsilon$ and $S$ would shift the phase transition temperature, and possibly slightly shift the width in changing the degree of temperature-dependence of the field. However, changing these parameters would not change the strength of the intermolecular interactions, which is more responsible for shifting the width of the phase transition, as shown in Figure S24. 

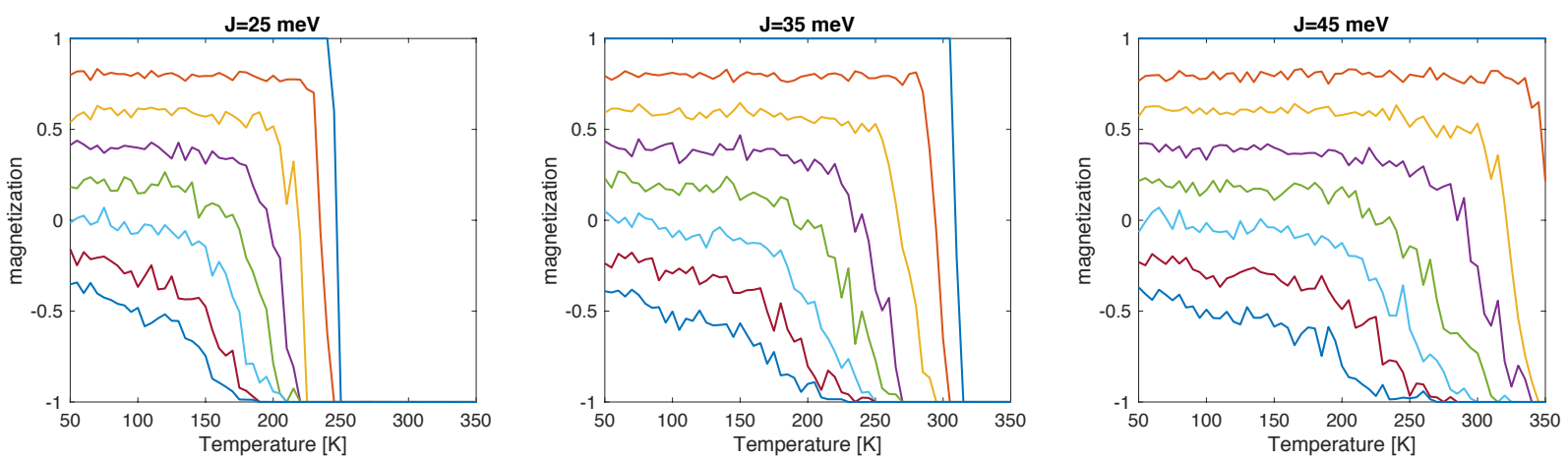

Figure S23: Role of changing J on the phase transition behavior (steps $=1 \mathrm{e} 5, \boldsymbol{\epsilon}=109.5 \mathrm{meV}$, $\left.\mathrm{S}=14.6 \mathrm{k}_{\mathrm{B}}, \mathrm{J}=35 \mathrm{meV}\right)$.

Changing the intermolecular coupling in this case both changes the phase transition temperature, as shown in describing the temperature-dependent field above. $\mathrm{J}=35 \mathrm{meV}$ was chosen as the coupling that most closely matches the experimental values.

For the Ising model runs shown in Figure 7 of the main text, we can determine the width and temperature of the phase transition using similar fits as were used in the optical data. Using the following equation, where $\chi_{\mathrm{ODPA}}$, corresponds to the mole fraction of the ligand shell, we can simplify the fit to the data shown in Figure 7 to two parameters.

$$
\text { Fraction ordered phase }=\chi_{\mathrm{ODPA}}\left(1-\tanh \left(\frac{T-T_{P T}}{\Delta T}\right)\right)-1
$$

\begin{tabular}{|l|l|l|l|l|l|l|l|l|}
\hline $\begin{array}{l}\text { Fraction } \\
\text { ODPA }\end{array}$ & 1 & .9 & .8 & .7 & .6 & .5 & .4 & .3 \\
\hline $\mathrm{T}_{\mathrm{PT}}$ (error) & $309(.1)$ & $293(.4)$ & $271(1)$ & $248(2)$ & $225(2)$ & $204(2)$ & $182(3)$ & $159(4)$ \\
\hline$\Delta \mathrm{T}$ (error) & $2.3(.2)$ & $5.5(.7)$ & $12.9(2)$ & $21.0(3)$ & $29.7(3)$ & $34.6(4)$ & $41.6(6)$ & $51.8(7)$ \\
\hline
\end{tabular}

Table S4: Fitting parameters for the model 


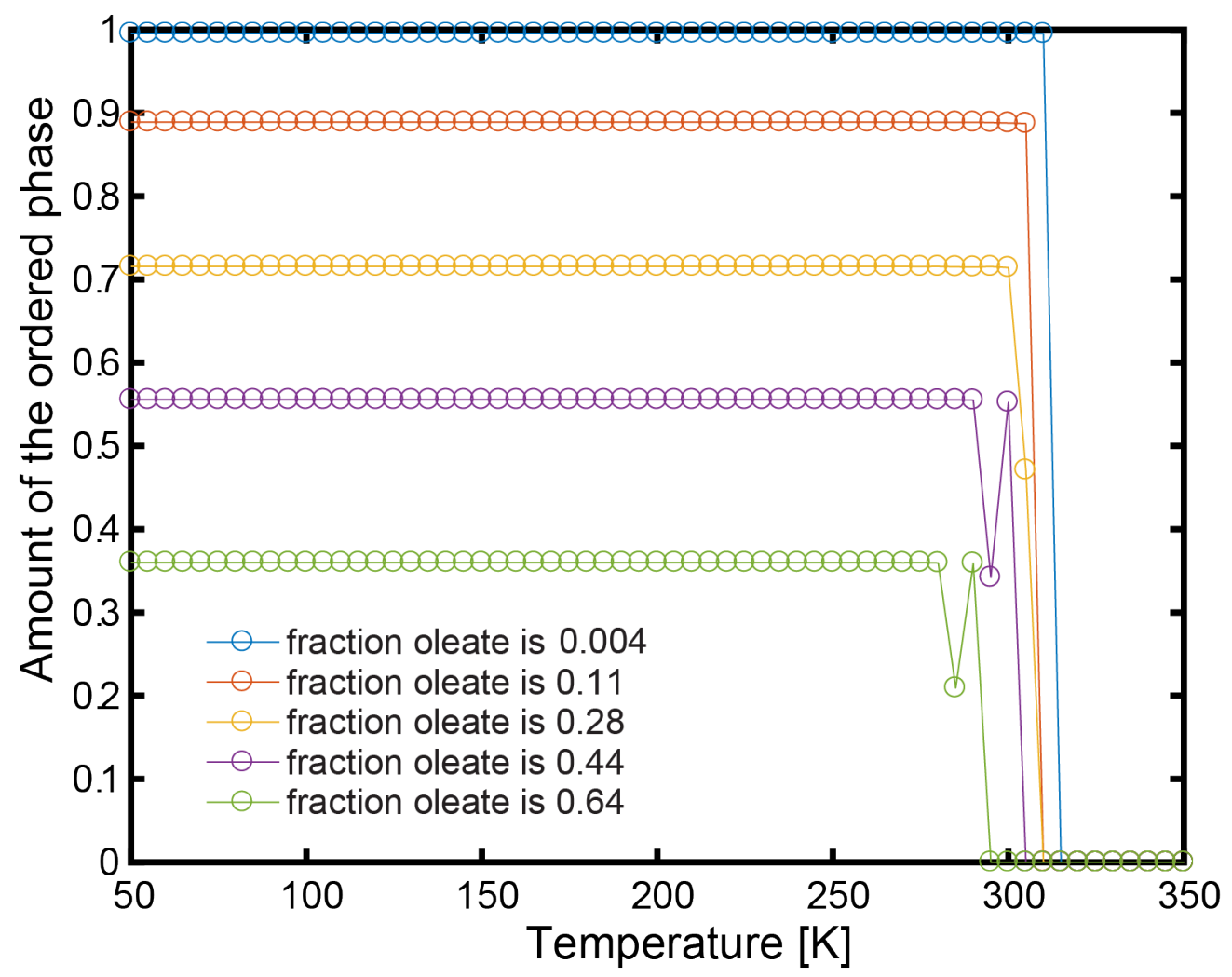

Figure S24: Phase transition behavior assuming a single square domain of oleate is present. All other parameters are the same as in previous simulations ( $\mathrm{steps}=1 \mathrm{e} 7, \mathrm{e}=109.5, \mathrm{~S}=14.6 \mathrm{k}_{\mathrm{B}}, \mathrm{J}=35$ $\mathrm{meV})$.

\section{Additional experimental methods}

Temperature-dependent time-resolved photoluminescence:

Measurements were performed using a time-correlated single photon counting apparatus. This consisted of a Picoquant Fluotime 300 spectrometer, a PMA 175 detector, and the LDH-P-C-405 diode laser with a $407 \mathrm{~nm}$ excitation, and a pulse width of approximately $50 \mathrm{ps}$. The repetition rates of the laser were tuned between $2 \mathrm{MHz}$ and $5 \mathrm{MHz}$ depending on the QD lifetime. For each pulse the maximum pulse power is less than $0.3 \mathrm{~nJ}$, with a spot size on the order of 100 micron in diameter, resulting in $<<1$ excitons created per QD per pulse. The temperature was modulated using a Lakeshore 330 temperature controller with the sample placed inside a Janis STVP-100 continuous-flow cryostat. Samples were prepared by dissolving a small amount of QDs in 2,2,4,4,6,8,8-heptamethylnonane. The sample cell used consisted of two sapphire windows separated by a Ti-coated Al spacer. PL transients were fit to monoexponential functions over the first decade and a half of the decay.

\section{References}


(S1) Balan, A. D.; Eshet, H.; Olshansky, J. H.; Lee, Y. V.; Rabani, E.; Alivisatos, A. P. Effect of Thermal Fluctuations on the Radiative Rate in Core/Shell Quantum Dots. Nano Lett. 2017, 17, 1629-1636.

(S2) Olshansky, J. H.; Balan, A. D.; Ding, T. X.; Fu, X.; Lee, Y. V.; Alivisatos, A. P. Temperature-Dependent Hole Transfer from Photoexcited Quantum Dots to Molecular Species: Evidence for Trap-Mediated Transfer. ACS Nano 2017, 11, 8346-8355.

(S3) Mendelsohn, R.; Davies, M. A.; Brauner, J. W.; Schuster, H. F.; Dluhy, R. A. Quantitative Determination of Conformational Disorder in the Acyl Chains of Phospholipid Bilayers by Infrared Spectroscopy. Biochemistry 1989, 28, 8934-8939.

(S4) Badia, A.; Cuccia, L.; Demers, L.; Morin, F.; Lennox, R. B. Structure and Dynamics in Alkanethiolate Monolayers Self-Assembled on Gold Nanoparticles: A DSC, FT-IR, and Deuterium NMR Study. J. Am. Chem. Soc. 1997, 119, 2682-2692. 